\title{
The RNA helicase DHX30 coordinates cytoplasmic translation and mitochondrial function contributing to cancer cell survival
}

\author{
Bartolomeo Bosco ${ }^{1}$, Annalisa Rossi ${ }^{1}$, Dario Rizzotto ${ }^{1}$, Sebastiano \\ Giorgetta $^{1}$, Alicia Perzolli ${ }^{1}$, Francesco Bonollo ${ }^{1}$, Angeline Gaucherot ${ }^{2}$, \\ Frédéric Catez ${ }^{2}$, Jean-Jacques Diaz ${ }^{2}$, Erik Dassi ${ }^{1 \#}$, Alberto Inga ${ }^{1 \#}$
}
${ }^{1}$ Department of Cellular, Computational and Integrative Biology, CIBIO, University of Trento, via Sommarive 9, 38123, Trento, Italy
${ }^{2}$ Inserm U1052, CNRS UMR5286 Centre de Recherche en Cancérologie de Lyon, F-69000 Lyon, France. Centre Léon Bérard, F-69008 Lyon, France, Université de Lyon 1, F-69000 Lyon, France

\#corresponding authors:

erik.dassi@unitn.it

alberto.inga@unitn.it

Running title: DHX30 controls global translation

Keywords: DHX30, translation efficiency, polysomal profiling, mitoribosome, ribosome biogenesis 


\begin{abstract}
DHX30 was recently identified as a critical element of an RBP complex participating in translational control of mRNAs containing a target 3'UTR cis element, in the context of p53dependent apoptosis. Here we show that DHX30 exhibits a more general, housekeeping function that comprises a combination of activities exerted by an isoform expressed in the cytoplasm and one, more abundant, localized in the mitochondria. Stable HCT116 cells depleted of both DHX30 isoforms showed constitutive changes in the repertoire of mRNAs associated with polysomes and enhanced translation of mRNAs coding for cytoplasmic ribosomal proteins. On the contrary, nuclear-encoded mitoribosome transcripts showed reduced translation efficiency in DHX30-depleted cells. Furthermore, HCT116 shDHX30 cells exhibited increased rRNA synthesis, higher amounts of ribosomes, and increased global translation. Surprisingly, they also showed reduced proliferation, which was also observed upon transient silencing of just the DHX30 cytoplasmic transcript. HCT116 DHX30-depleted cells showed reduced mitochondrial energy metabolism, based on oxygen consumption rate, but did not show evidence of compensatory glycolysis. Impaired mitochondrial function was also related to decreased expression of mitochondrially encoded OXPHOS components. Results were extended to U2OS and MCF7 cell lines. A gene signature comprising DHX30 and fourteen mitoribosome transcripts that are candidate direct DHX30 targets showed prognostic value in distinct cancer types from TGCA data, with higher expression being associated to reduced overall survival. Hence, it appears that DHX30 contributes to cell homeostasis through the coordination between ribosome biogenesis, global translation, and mitochondrial functions. Targeting DHX30 could, thus, expose a vulnerability in cancer cells.
\end{abstract}




\section{Introduction}

The RNA binding protein (RBP) DHX30 is an ATP-dependent RNA helicase highly expressed in neural cells and somites during embryogenesis in mice. It plays an important role in development and its homozygous deletion is lethal for embryos ${ }^{1}$. In humans, DHX30 is involved in the antiviral function of the zinc-finger ZAP protein. Indeed, it was demonstrated that these two proteins directly interact with each other via the N-terminal domain and DHX30 is necessary for the optimal antiviral activity of ZAP ${ }^{2}$. Recently, six de novo missense mutations were identified in DHX30 in twelve unrelated patients affected by global developmental delay (GDD), intellectual disability (ID), severe speech impairment and gait abnormalities ${ }^{3}$. All mutations caused amino acid changes in the highly conserved helicase motif, impairing protein's ATPase activity or RNA recognition. Moreover, cells that carry these mutations in DHX30 exhibit an increased propensity to trigger stress granules (SG) formation decreasing global translation ${ }^{3}$. It was shown that DHX30 could play an important role also in human fibroblast and osteosarcoma mitochondria. Indeed, in that compartment it interacts with a Fas-activated serine-threonine kinase (FASTKD2), modulating the mitochondrial ribosome maturation and assembly 4 . Our group and collaborators recently compared p53-mediated responses to Nutlin-3 treatment in three different cancer cell lines: HCT116, SJSA1 and MCF7 ${ }^{5,6}$. It was observed that 553 activation caused cell cycle arrest in HCT116, massive apoptosis in SJSA1 and an intermediate phenotype in MCF7, consistent with earlier reports ${ }^{7}$. By analyzing the cell lines' translatomes (i.e.transcripts loaded on polysomes and in active translation), we discovered that only the $0.2 \%$ of the genes were commonly differentially expressed (DEGs). Nevertheless, the polysome profiling of SJSA1 revealed an enrichment of proapoptotic transcripts ${ }^{6}$ in the fraction corresponding to polysomes, that featured instances of a specific cis-element, labeled as CGPD, standing for CG-rich motif for p53-dependent death, in their 3'UTRs.

The DExH box RNA helicase DHX30 was found as one RBP whose binding to the motif correlated with a specific cell fate. Indeed, DHX30 expression was expressed at higher levels in HCT116 than SJSA1, resulting in a lower translation of GCPD-motif-containing transcripts in HCT116 cells. Depletion of DHX30 expression in HCT116 by stable shRNA increased the translation potential of mRNAs containing the CGPD motif, resulting in higher levels of apoptosis after p53 activation by Nutlin treatment, mimicking in part the apoptotic phenotype of SJSA1 ${ }^{6}$.

In this work, we focused on a wider characterization of the phenotypic role of DHX30 in the colorectal cancer cell line HCT116. By virtue of two alternative promoters leading to the production of a cytoplasmic or a mitochondrial isoform, DHX30 couples mitochondrial function, ribosome biogenesis and global translation, while inhibiting translation of CGPD-containing mRNAs pro-apoptotic targets in the context of p53-dependent apoptosis ${ }^{6}$. Depleting DHX30 in HCT116 cells reduced cell proliferation and sensitised cells to small molecules targeting mitochondrial function.

\section{Results}

Silencing DHX30 enhances translation in HCT116 cells

We previously identified DHX30 as a negative modulator of p53-dependent apoptosis, performing polysomal profiling followed by RNA-seq of HCT116 cells clones stably depleted for DHX30 and treated with the MDM2 inhibitor Nutlin-3 ${ }^{7}$. Here we reanalyzed this dataset (GSE95024), complementing it by performing a total RNA profiling of the same cells by RNA-seq (GSE154065), and focusing on the comparison between DHX30-depleted (shDHX30) and control (shNT) HCT116 cells in the untreated control.

We compared the gene expression changes at the polysomal or total cytoplasmic RNA levels and performed a gene set enrichment analysis ${ }^{8}$. This revealed an increased abundance of ribosomal proteins and ribosome biogenesis genes in shDHX30 cells only at the polysomal level (Figure 1A, Table S1). Consistently, analysis of translation efficiency (TE), measured as the ratio between relative RNA abundance in polysomal and total cytoplasmic fractions, revealed that DHX30 depletion is associated with higher TE for transcripts coding for ribosomal protein subunits (RPL, RPS, Figure 1B). On the other 
end, nuclear transcripts coding mitochondrial ribosomal proteins exhibited reduced TE in DHX30depleted cells, despite showing higher constitutive TE compared to the cytoplasmic ribosomal transcripts (mRPL, mRPS, Figure 1B). A trend for higher expression of these transcripts in DHX30-depleted cells was also apparent (Figure S1).

We thus sought to understand if DHX30 could be directly regulating this class of genes and exploited the data of DHX30 eCLIP assay performed in K562 cells as part of the ENCODE project ${ }^{9}$. Despite the different cell line, the eCLIP data indicate that DHX30 can bind 67 ribosomal (36 RPLs and 31 RPSs) and 23 mitoribosomal (16 MRPLs and 7 MRPSs) protein transcripts (Table S1) ${ }^{9}$.

Consistent with the higher TE for RPL and RPS transcripts, DHX30 depletion in HCT116 cells was associated with higher relative amounts of ribosomes extracted and quantified from a sucrose cushion (Figure 1C), and higher rRNA levels (Figure 1D, E). Furthermore, DHX30-depleted cells not only showed evidence of increased ribosome biogenesis, but also of higher global translation (Figure 1F, 1G). Moreover, DHX30 protein was found associated with ribosomal subunits, 80S monosomes as well as with low molecular-weight polysomes in HCT116, MCF7 and U2OS cells (Figure S1B,C).

Hence, DHX30 appears to negatively influence ribosome biogenesis, global translation and also the translation of specific mRNAs. It is important to emphasize that these are not necessarily dissociated events, as a change in ribosome number could be sufficient to impact on translation specificity ${ }^{10}$. 
a

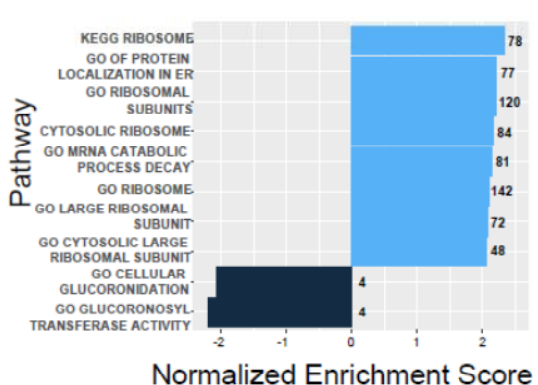

d

$\square$ HCT_shNT $\square$ HCT_shDHX30

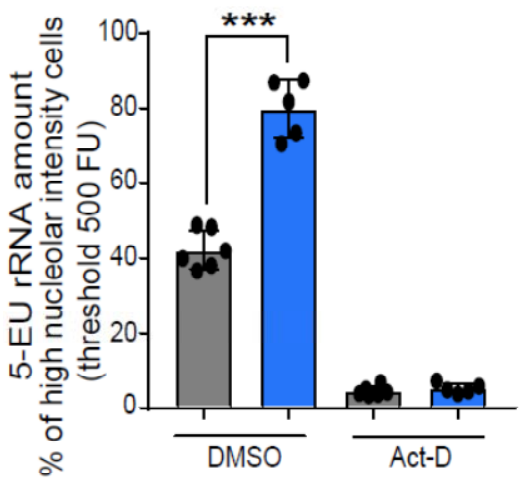

f

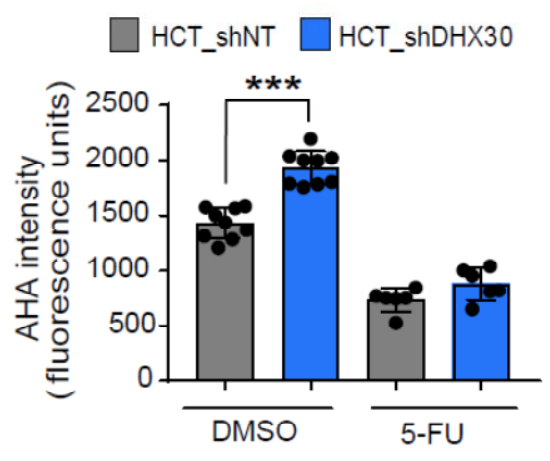

b

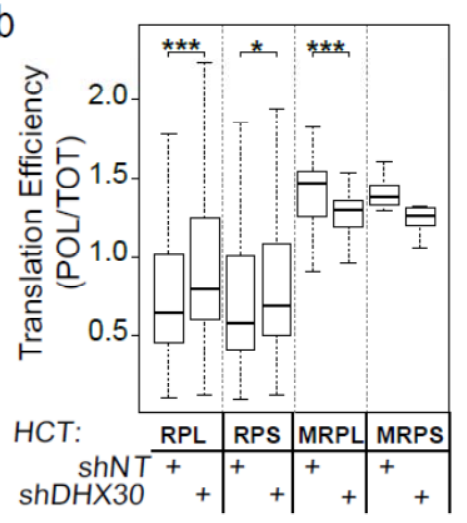

e
C

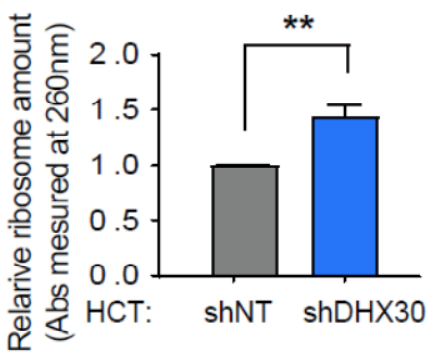

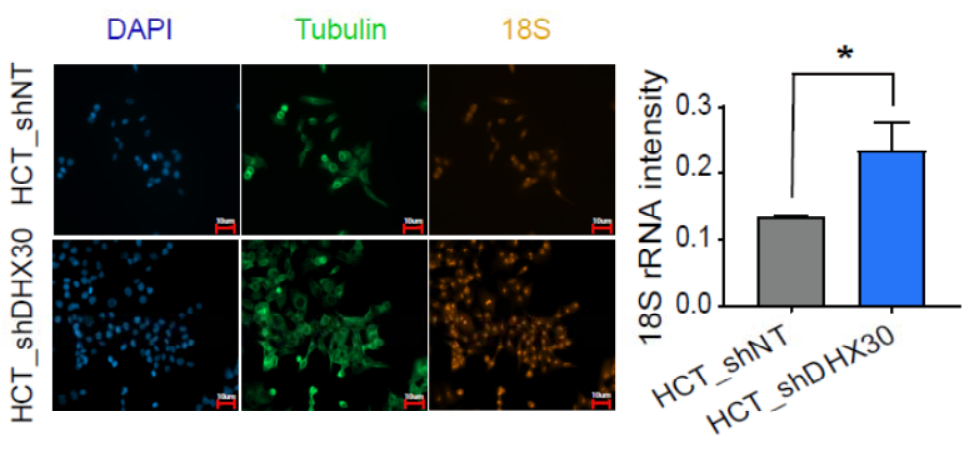

g

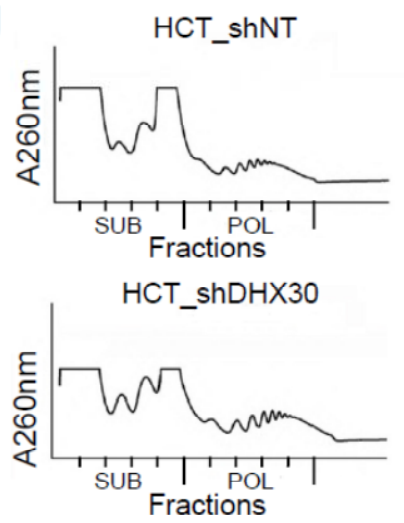

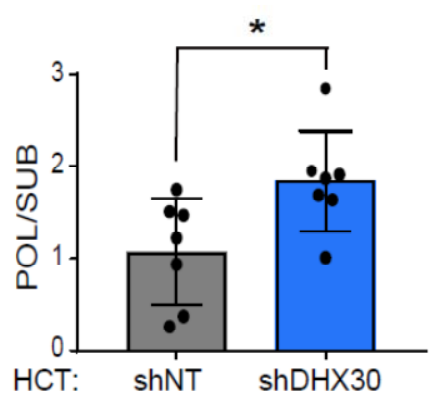

Figure 1. DHX30 reduces ribosome biogenesis and translation in HCT116 colorectal cancer cells.

A) Most significant terms by Gene Set Enrichment Analysis of the polysome-bound DEGs (HCT_shDHX30 vs HCT sh_NT). Bars plot the Normalized Enrichment Score, (NES). Numbers indicate the number of genes in the leading edge. See Table S1 for full results. B) Box plot of Translation efficiency for the indicated transcript groups. The RNA-seq CPM in polysomal over total RNA was measured. Results obtained in HCT116_shDHX30 cells are compared to the shNT control clone. C) Amounts of ribosomes produced in HCT116_shDHX30 and HCT_shNT. After ribosomes isolation, protein absorbance was measured at $\lambda 260 \mathrm{~nm}$. Data are normalized on shNT and are mean $\pm \mathrm{SD}(\mathrm{n}=3) ; * \mathrm{p}<0.05$. D) Amount of ribosomal RNA estimated based on the fluorescence intensity of 5-ethynyl uridine (5-EU) incorporated in nascent rRNAs present in the nucleoli. HCT116_shDHX30 cells are compared to the shNT control clone in untreated condition while Actinomycin-D treatment was used as a control. Data are mean \pm $\mathrm{SD}(\mathrm{n}=6) ; * * * \mathrm{p}<0.001$. E) (Left) Representative images of one of three independent experiments of Fluorescent In Situ Hybridization (FISH) for rRNA precursor 18S (red). Immunofluorescence of tubulin (green) and staining with DAPI (blue) were used to visualize cells. (Right) Box plot of $18 \mathrm{~S}$ rRNA intensity quantification comparing HCT116_shDHX30 with HCT_shNT analyzed with Cell Profiler software. Data are expressed as mean per well \pm $\mathrm{SD}$ of three biological replicates in which 10 nuclei were quantified for each replicate; ${ }^{*} \mathrm{p}<0.05$. F) Analysis of 
global translation based on the fluorescence intensity of L-azidohomoalanine (AHA) incorporated in nascent proteins present in the cytoplasm. HCT116_shDHX30 cells are compared to the shNT control clone in untreated condition while 5-Fluorouracil treatment was used as positive control. Data are mean $\pm \mathrm{SD}$ ( $\mathrm{n}=6$ to 9 ); ***p $<0.005$. G) (Left) Polysome profiling of HCT_shDHX30 and HCT_shNT, revealed by the measurement of absorbance at a wavelength of $260 \mathrm{~nm}$. (Right) Box plot of the relative quantification of the area under the curve of fractions corresponding to the polysomes related to the fractions corresponding to the ribosomal subunits and 80S monosome (POL/SUB, an estimate of translation efficiency). Data are mean $\pm \mathrm{SD}(\mathrm{n}=7) ;{ }^{*} \mathrm{p}<0.05$.

\section{DHX30 depletion alters mitochondrial function via multiple mechanisms}

The impact of DHX30 depletion on the expression and translation efficiency of nuclear-encoded mitoribosomal proteins caught our attention, as it showed the opposite trend compared to ribosomal protein transcripts (Figure 1B, Figure S1). First, we established the potential for DHX30 to bind to mitoribosomal protein transcripts by RIP assays, choosing MRPL11 (UL11m), that was previously evaluated in cancer cells ${ }^{3}$, and MRPS22 (mS22), as a representative of the large and small subunits, respectively (Figure 2A). Next, we observed that DHX30-depleted cells showed reduced expression of these two genes at both the RNA and protein levels (Figure 2B, C). These findings suggest that DHX30 directly promotes stability and/or translation of mitoribosome transcripts. The modulation of mitoribosome proteins expression might impact on mitochondrial translation and contribute to the functions described for DHX30 in the mitochondrial matrix ${ }^{4,11}$. Notably, the DHX30 gene features two promoters, and the transcript resulting from the internal (more $3^{\prime}$ ) one includes an alternative first exon that contains a predicted mitochondrial localization signal. Mitochondrial localization of the protein was confirmed by immunofluorescence (Figure 2D), confirming previous results in human fibroblasts ${ }^{4}$ and APEX-seq data ${ }^{12}$. We checked the relative levels of the DHX30 transcripts from the two promoters and found the putative mitochondrial isoform to be about four times more abundant (Figure 2E), as indicated also by western blot analysis on extracts of mitochondrial and cytoplasmic fractions (Figure 2F).

Stable DHX30-silencing was obtained using shRNAs that targeted both cytoplasmic and mitochondrial transcripts (Figure S2A). To confirm these results, we employed transient silencing using one siRNA specific for cytoplasmic DHX30 (from here on labeled cDHX30, hence called siDHX30-C) or an siRNA targeting transcripts from both promoters (cDHX30 and mDHX30, hence called siDHX30$\mathrm{C}+\mathrm{M})$. The activity and specificity of the two siRNAs was assessed by qRT-PCR 48 hour and 96 hours post-silencing (Figure S2B, S2C). Transient silencing of both cytoplasmic and mitochondrial DHX30 isoforms (siDHX30-C+M) resulted in a reduction of the expression of MRPL11 and MRPS22 (Figure 2G, H) similar to waht we previously observed with stable silencing of both isoforms (Figure 2C). The silencing of cytoplasmic DHX30 only (siDHX30-C) resulted in a weaker effect. This indicates that both DHX30 variants have to be depleted to strongly impact on mitoribosome gene expression. The DHX30 transient silencing was extended to U2OS, osteosarcoma-derived cells that express relatively high levels of DHX30, and to MCF7, a breast cancer derived cell line (Figure S2D, S2E). Only for MCF7, the depletion of DHX30 obtained by the siDHX30-C+M was associated with a reduction in the expression of MRPL11 and MRPS22 mRNAs (Figure S3). 


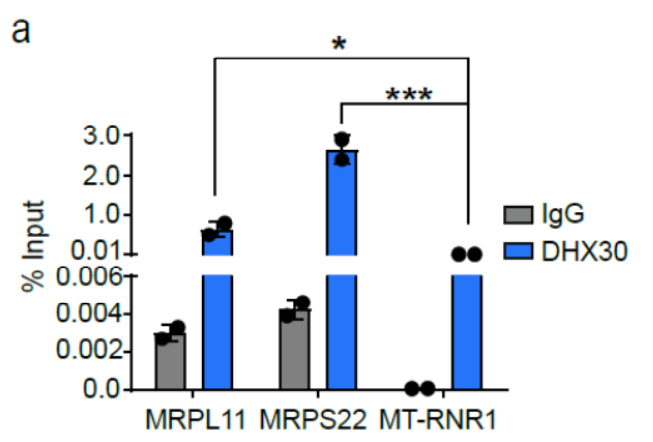

b

C
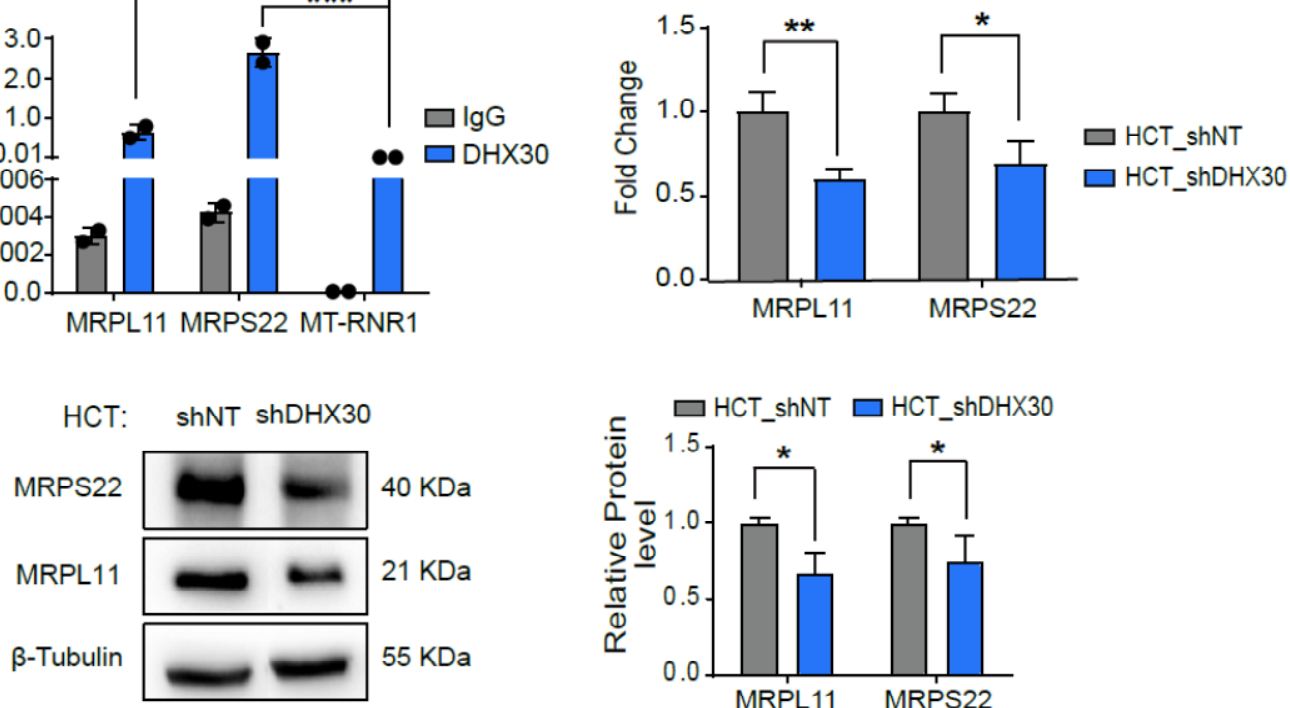

d
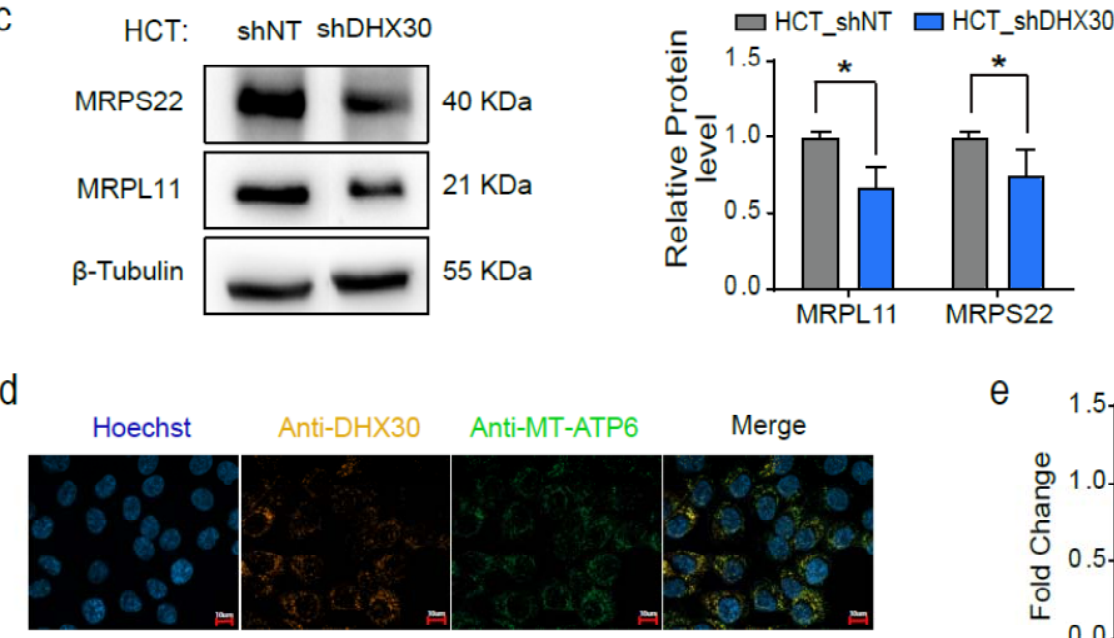

d
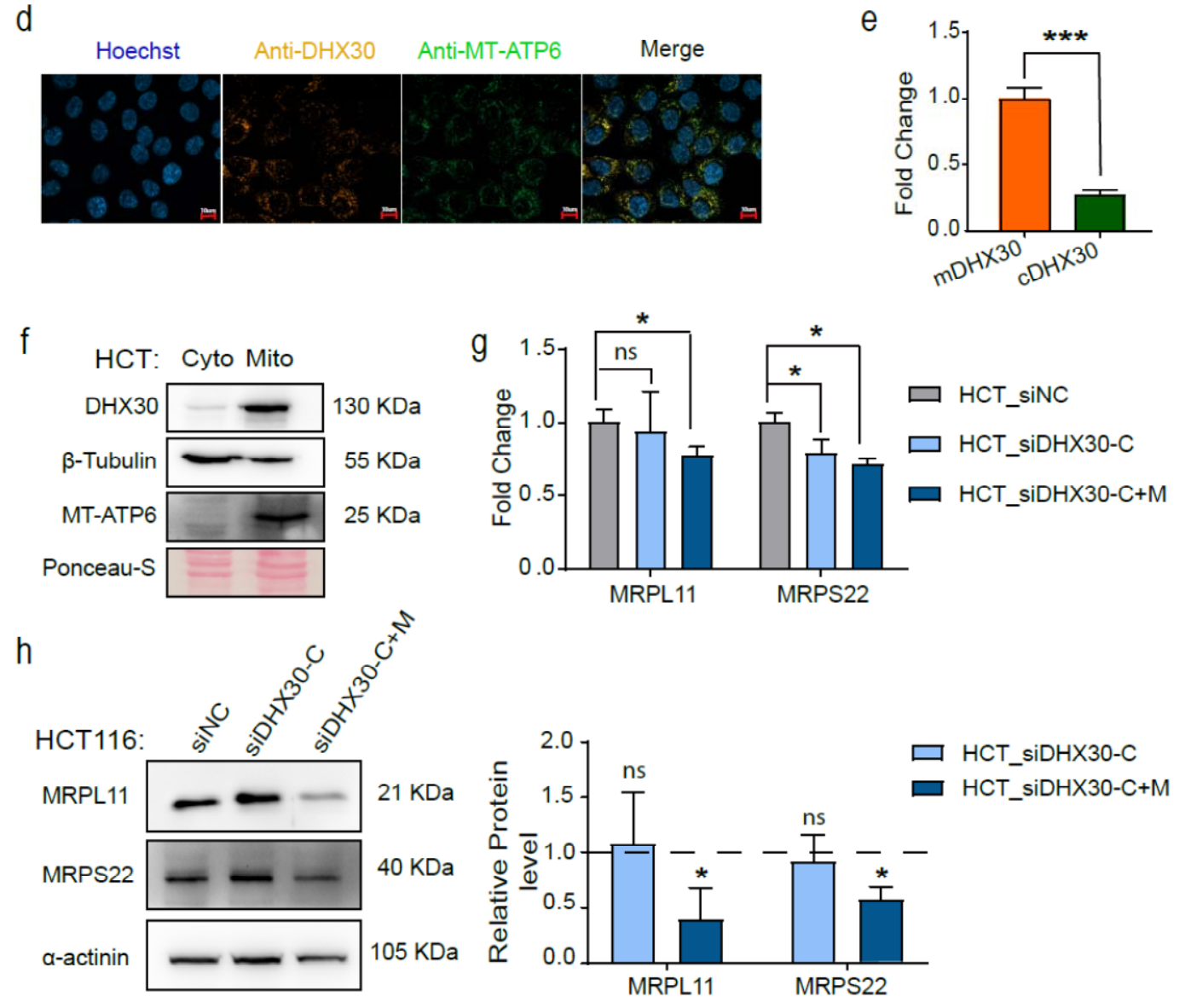

Figure 2. Cytoplasmic DHX30 modulates the expression of nuclear encoded mito-ribosome components MRPL11 and MPRS22.

A) RNA immunoprecipitation experiments to study the binding of DHX30 to MRPL11 and MRPS22 transcripts. Results obtained with a primary antibody targeting DHX30 (blue) or the IgG control (grey) are plotted relative as \% of input. Data are mean and individual points $(\mathrm{n}=2)$; ** $<0.01$. B) Relative mRNA levels of MRPL11 and MRPS22 in HCT116_shDHX30 compared to the shNT control clone. Data are mean $\pm \mathrm{SD}(\mathrm{n}=3)$; *p < 0.05; ** $\mathrm{p}<$ 
0.01. C) (Left) Protein levels of MRPL11 and MRPS22. $\beta$-Tubulin was used as loading control. Immunoblots represent one of three independent experiments. (Right) MRPL11 and MRPS22 protein quantification in HCT116_shDHX30 normalized on shNT control clone. Data are mean \pm SD $(n=3) ; * p<0.05$. D) Representative images of one of three independent immunofluorescence experiments to colocalize DHX30 (red) with MT-ATP6 (green, used as mitochondrial marker). Staining with Hoechst (blue) was used to visualize cells' nuclei. E) Relative mRNA levels of cytoplasmic DHX30 (cDHX30) and the mitochondrial variant (mDHX30, set to 1). Data are mean $\pm \mathrm{SD}(\mathrm{n}=3) ; * * * \mathrm{p}<0.001$. F) Relative DHX30 protein levels in cytoplasm and mitochondria. $\beta$-Tubulin and MTATP6 are used as controls of cytoplasmic-mitochondrial fractionation and Ponceau-S staining is used as loading control. One of three independent experiments is shown. G) mRNA levels of MRPL11 and MRPS22 in HCT116 silenced transiently for cytoplasmic DHX30 (HCT_siDHX30-C) or for both cytoplasmic and mitochondrial variants (HCT_siDHX30-C+M). Data are compared to the siRNA negative control (HCT_siNC) and are mean \pm SD ( $\mathrm{n}=3$ ); $* \mathrm{p}<0.05$. H) (Left) MRPL11 and MRPS22 protein levels in HCT116 silenced for cytoplasmic DHX30 (HCT_siDHX30-C) or for both cytoplasmic and mitochondrial variants (HCT_siDHX30-C+M). Immunoblot represents one of three independent experiments. (Right) MRPL11 and MRPS22 protein quantification in HCT116_siDHX30-C and HCT116_siDHX30-C+M normalized on $\alpha$-actinin and compared to siNC control. Data are mean $\pm \mathrm{SD}(\mathrm{n}=3) ; * \mathrm{p}<0.05$.

We next focused on the expression of mitochondrially encoded genes. First of all, we established that DHX30 depletion did not impact on the number of mitochondrial genomes by digital PCR (Figure 3A). Instead, the expression of most mitochondrially encoded transcripts was reduced (Figure 3B). The steady-state expression of two mitochondrially encoded proteins, MT-ATP6 and MT-ATP8, was shown to be reduced in HCT116_shDHX30 cells (Figure 3C, S3E). We pursued the same analysis after transient silencing, confirming the lower expression of mitochondrially encoded genes at the RNA and protein levels, but only when the DHX30 mitochondrial variants (siDHX30-C+M) was silenced and analysed 96 hours post silencing (Figure 3D, E). In fact, although DHX30 depletion was visible already after 48 hours (Figure S2B) from the addition of the siRNAs, more time was needed to appreciate a reduction in the expression of mitochondrially encoded genes. The expression of mitochondrially encoded genes was checked also after transient DHX30 silencing in U2OS and MCF7 cells (Figure S4). In U2OS none of the seven tested transcripts were down-modulated by DHX30 silencing, regardless of the siRNA used. In fact, three of them were slightly upregulated. MT-ATP6 was also examined by western blot and no differences in amount was apparent (Figure S4A, S4C). Instead, DHX30 depletion (by siDHX30 $\mathrm{C}+\mathrm{M}$ ) in MCF7 led to a significant reduction in the expression of all tested mitochondrial transcripts, although for MT-ATP6 the reduction was not confirmed at protein levels (Figure S4B, S4D).

Finally, we evaluated markers of carbon metabolism and mitochondrial respiration capacity by an Agilent SeahorseXF real-time analyzer. HCT116_shDHX30 cells had a significantly lower basal oxygen consumption rate compared to the control cell line. Notably, DHX30-depleted cells did not show compensatory glycolysis in basal culture condition, not even when we chemically abolished mitochondrial respiration. We indeed observed a lower compensatory glycolysis compared with the control (Figure 3F). These results suggest that HCT116_shDHX30 cells do not show a typical Warburg effect ${ }^{13}$, and are expected to produce less energy due to reduced mitochondrial respiration.

Collectively, the depletion of DHX30 seems to generate an imbalance in cell homeostasis, with higher demand of chemical energy from ribosome biogenesis and cytoplasmic translation but lower mitochondrial activity. 
a

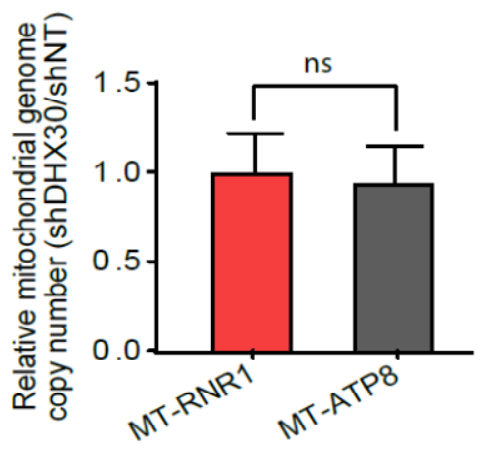

C

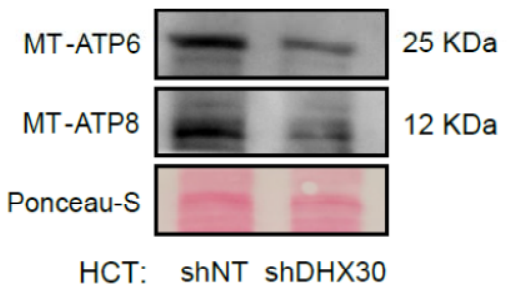

d

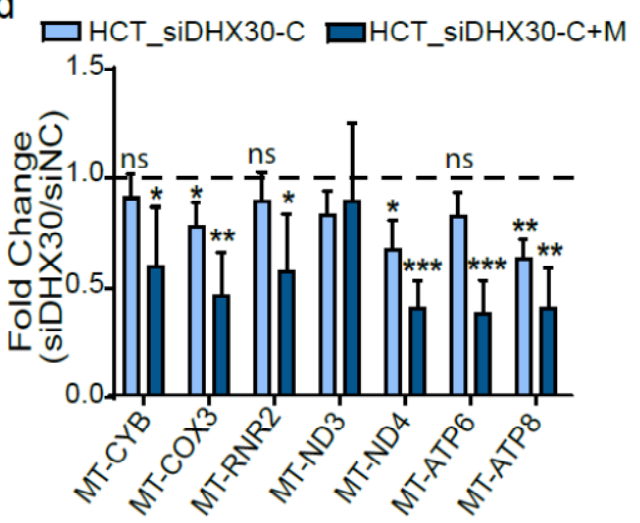

f

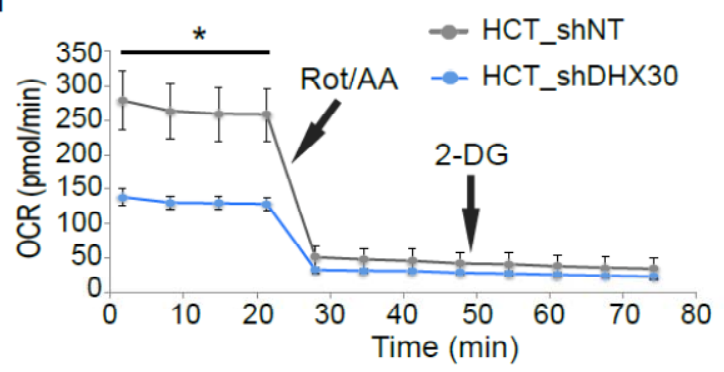

e b
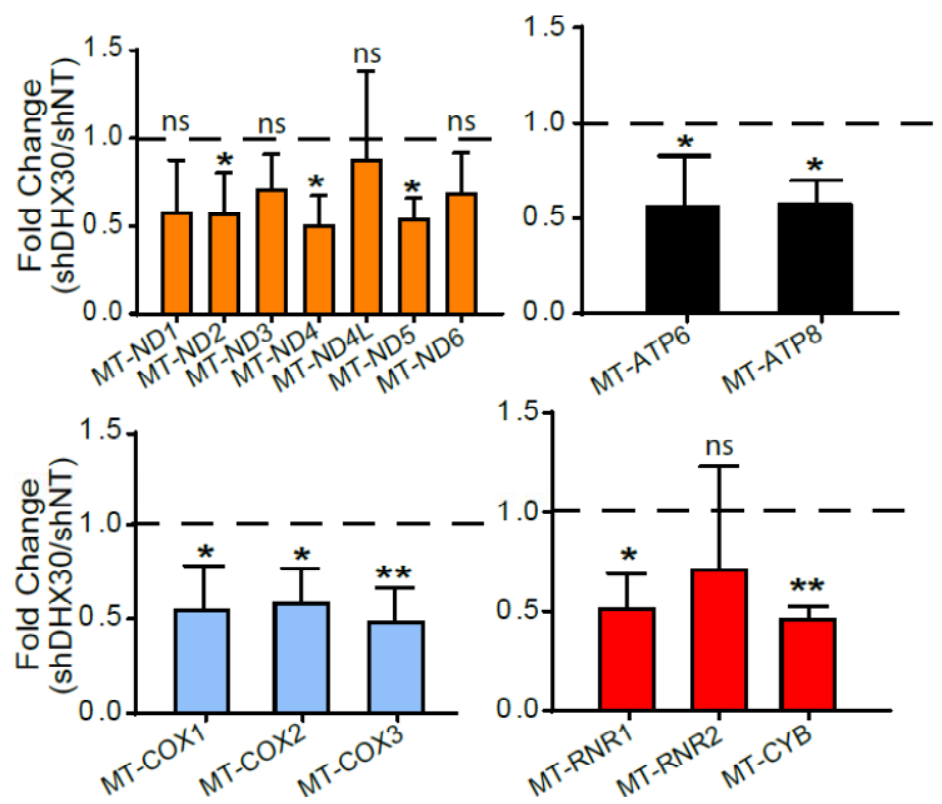
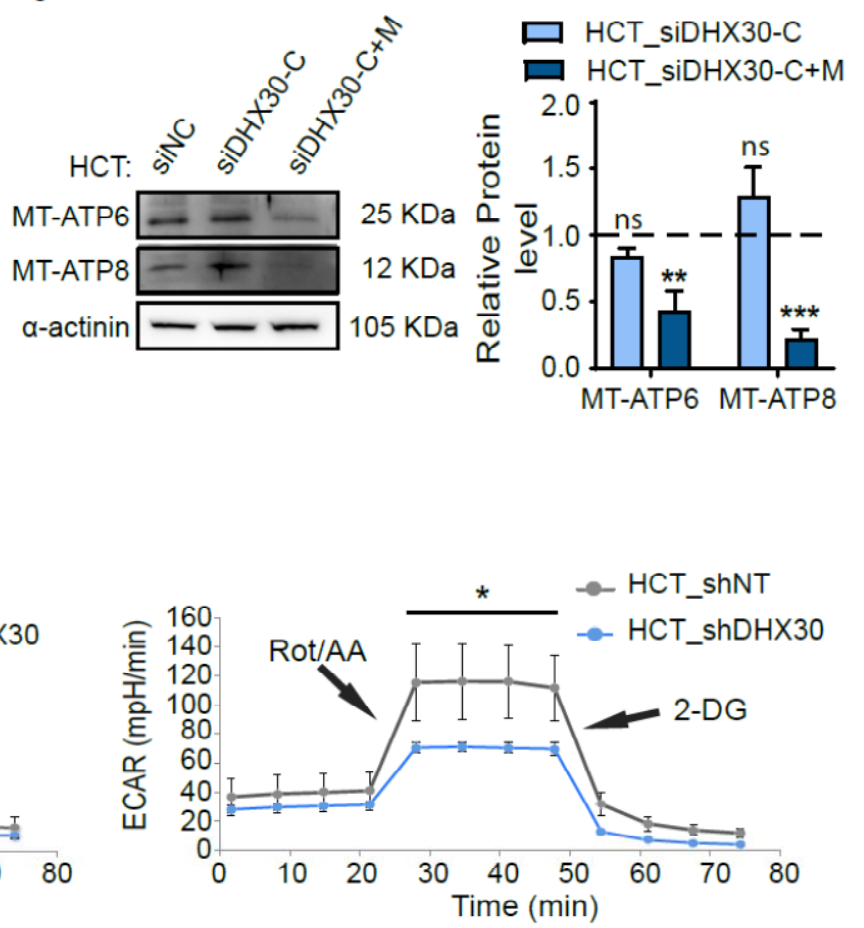

Figure 3. Depletion of DHX30 reduces the expression and function of mitochondrially encoded OXPHOS components.

A) Relative mitochondrial genome copy number in HCT116_shDHX30 and -shNT measured by droplet digital PCR. MT-RNR1 and MT-ATP8 were amplified along with the nuclear diploid marker gene CTDSP1. Bars plot mean $\pm \mathrm{SD}(\mathrm{n}=3)$. B) Relative mRNA levels of mitochondrially encoded OXPHOS components in HCT116_shDHX30 compared to HCT116_shNT (dashed line, set to 1). Data are mean \pm SD (n=3); *p < 0.05; **p $<0.01$. C) Protein levels of MT-ATP6 and MT-ATP8 in mitochondrial lysates of HCT116_shDHX30 and the shNT control clone. Ponceau-S was used as loading control. Immunoblots represent one of two independent qualitative comparisons. D) Relative mRNA levels of the indicated mitochondrially encoded OXPHOS components in HCT116 
transiently silenced for DHX30 expression (siDHX30-C, siDHX30-C+M) compared to siNC (dashed line, set to 1). Data are mean \pm SD $(n=3) ; * p<0.05 ; * * p<0.01 ; * * *<0.001$. E) (Left) Protein levels of MT-ATP6 and MTATP8 in HCT116 transiently silenced as in D). $\alpha$-actinin was used as loading control. Immunoblots represent one of three independent experiments. (Right) MT-ATP6 and MT-ATP8 protein quantification in HCT116_siDHX30-C and HCT116_siDHX30-C+M normalized on $\alpha$-actinin and compared to siNC control. Data are mean \pm SD ( $\mathrm{n}=3$ ); $* * \mathrm{p}<0.01 ; * * * \mathrm{p}<0.001$. F) (Left) Measurement of the oxygen consumption rate (OCR) to evaluate mitochondrial respiration by Seahorse XF analyzer. Points before the Rotenone/Antimycin-A (Rot/AA) treatment correspond to the basal mitochondrial respiration. (Right) Extracellular acidification rate (ECAR) measurement was used as a means to measure glycolysis. Points before and after the Rotenone/Antimycin-A (Rot/AA) treatment correspond respectively to basal and compensatory glycolysis -in response to the block of mitochondrial respiration-. 2Deoxyglucose (2-DG) is then used to block glycolysis. For both panels, one of three independent replicates is presented. Data are mean \pm SD ( $n=3$ wells in the Seahorse cartridge); $* \mathrm{p}<0.05$.

Silencing of DHX30 impaired cell proliferation rate and increased apoptosis proneness. Next, we characterized the phenotypic impact of DHX30 depletion in HCT116. Consistent with the lower mitochondrial oxygen consumption rate, HCT116_shDHX30 cells showed a significantly lower proliferation rate compared to the control cell clone. This was observed by colony formation assay and confirmed through time-course proliferation by cell count via high-content microscopy or using a RealTime Cell Analyzer xCELLigence (Figure 4A-C). Interestingly, we observed a reduction in proliferation after DHX30 silencing also in a spheroid assay (Figure 4D). The lower growth rate in DHX30 depleted cells cannot be attributed to an arrest in a particular phase of the cell cycle (Figure $\mathbf{4 E}$ ). A reduction in proliferation was confirmed also by cell counting upon transient silencing of cDHX30 and, particularly, of both cDHX30 and mDHX30 (Figure 4F). The impact on cell proliferation was also examined using transient silencing in U2OS and MCF7. Consistent with the impact observed on gene expression, only MCF7 cells showed a significant reduction in proliferation (Figure S4E).

Furthermore, DHX30 depletion was associated with increased apoptosis after 48 hours of Nutlin treatment, as expected by our previous results ${ }^{6}$, but also when cells were treated with the topoisomerase inhibitor doxorubicin and with FCCP, an agent that causes mitochondrial membrane depolarization (Figure 4G). 
a

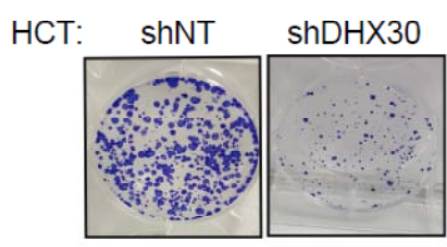

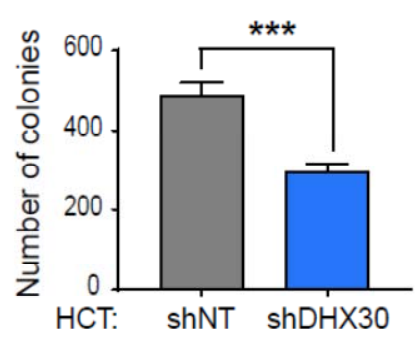

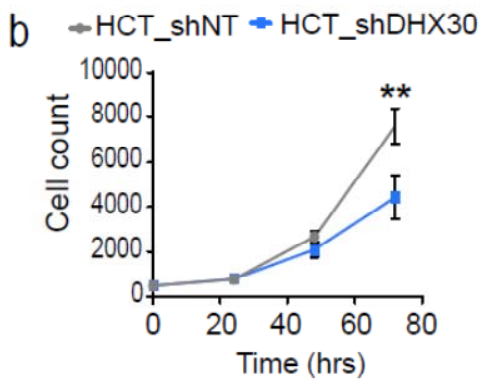

C

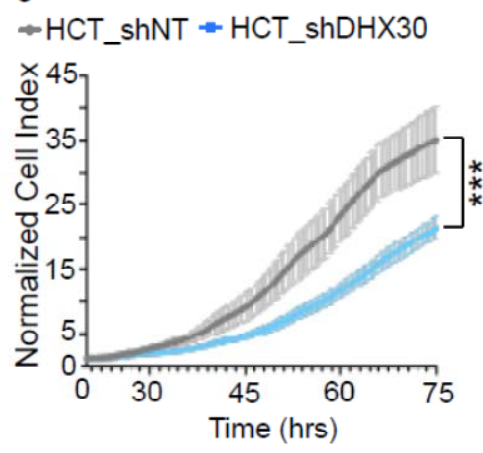

e

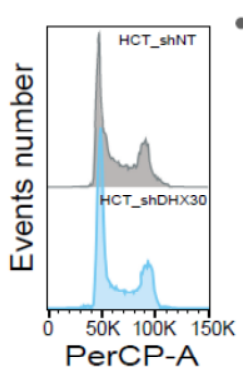

PerCP-A

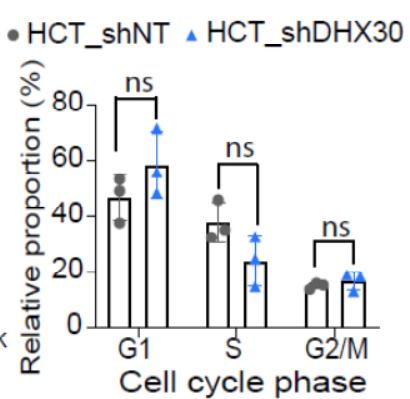

Cell cycle phase d Hours post seeding

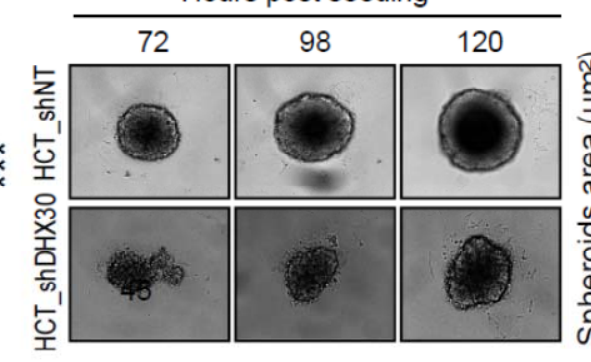

f
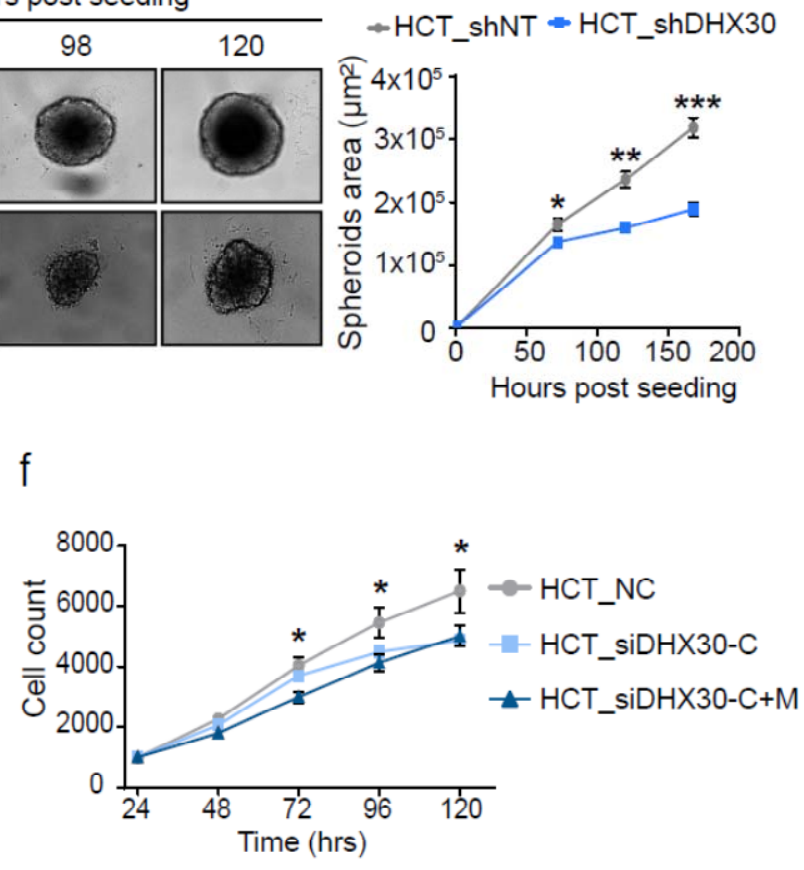

g

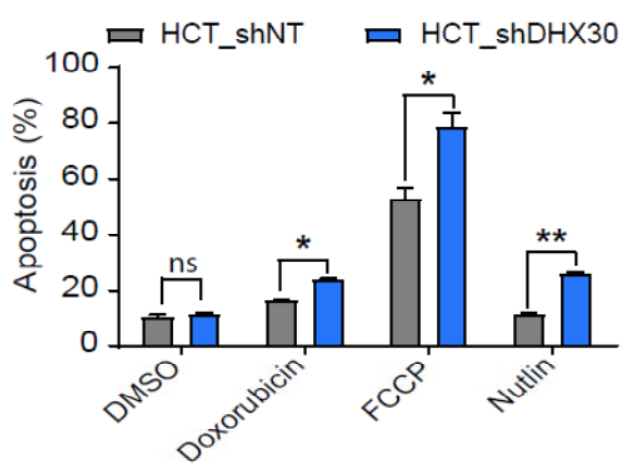

Figure 4. Depletion of DHX30 reduces proliferation and survival in basal and treatment conditions

A) (Left) Representative image of colony formation assays. (Right) Colony quantification by ImageJ software. Data are mean $\pm S D(n=3)$. B) Relative cell proliferation measured by high-content microscopy in digital phase contrast. Data are mean $\pm \mathrm{SD}(\mathrm{n}=3)$. C) Estimate of relative proliferation by an impedance-based Real-Time Cell Analyzer. Data are mean $\pm S D(n=3)$. D) Spheroid formation and growth assay. (Left) A representative image at the indicated time points is shown in the left panel. (Right) Spheroid area measured by ImageJ software. Data are mean \pm SD $(\mathrm{n}=3)$. E) (Left) Representative image of HCT116_shNT and shDHX30 cell cycle profiles. (Right) Quantification of cell cycle profile in HCT116 DHX30 depleted cells compared to shNT control. Data are mean \pm SD $(n=3)$. F) 
Relative proliferation measured as in B) but starting 24 hours after transient silencing DHX30 with the indicated siRNAs. G) Relative expression of the annexin-V apoptosis markers in cells treated for 48 hours with the indicated drugs or DMSO control. For all panels, data are mean $\pm \operatorname{SD}(n=3) ; * p<0.05 ; * * p<0.01$.

\section{DHX30 signature and cancer outcome}

Our results suggest that DHX30 exerts a constitutive function that improves cellular fitness by balancing energy metabolism and global translation potential. Furthermore, our previous study identified DHX30 as a negative modulator of the translation of specific mRNAs, thus controlling p53-dependent apoptosis ${ }^{6}$. Both these functions suggest that DHX30 could be a modifier of cancer cell properties potentially impacting on clinical variables.

Although total RNA-seq data are not a good proxy for investigating translation controls ${ }^{14}$, in this study we showed that DHX30 depletion impacts on steady-state levels of nuclear-encoded mitoribosomal transcripts. MRPL11 and, particularly, MRPS22 can be considered direct DHX30 targets. We next crossreferenced our TE and GSEA data with the RIP results and DHX30 eCLIP data in ENCODE ${ }^{9}$ and compiled a list of 14 mitoribosomal protein (MRP) transcripts considered DHX30 direct target candidates (Figure S5). Interrogating RNA-seq data of TGCA tumors through the GEPIA web resource ${ }^{15,16}$ the expression of DHX30, and of each MRP transcript or of the group of 14 candidate target MRPs appeared to be positively correlated in several cancer types (Figure S5A, S5B), including adrenocortical carcinoma, and hepatocellular carcinomas. No such correlation was instead apparent for other cancer types, including breast or colon adenocarcinomas. We next used this gene signature to stratify patients' clinical outcome. Interestingly, for the cancer types where a positive correlation in the expression of DHX30 and the 14 MRPs was observed, the combined 15-gene signature (i.e. including DHX30) showed a prognostic value. In particular, higher expression was associated with reduced Overall Survival or Disease-Free Survival (Figure 5A, B, Figure S5B-D). Instead, the comparison of DHX30 expression between tumors and matched controls did not show a consistent trend but confirmed a rather wide variation among samples, and tissue-specific effects including both cases of over-expression and downregulation (Figure S6). Finally, a pan-tissue view revealed a general positive correlation between the expression of ribosome and mitoribosome protein transcripts in normal samples, that is lost in cancer (Figure S7). These preliminary analyses suggest that a functional signature could be developed to predict aggressiveness in cancer types where DHX30 appears to stimulate mitoribosomal proteins expression. 


\section{ACC}

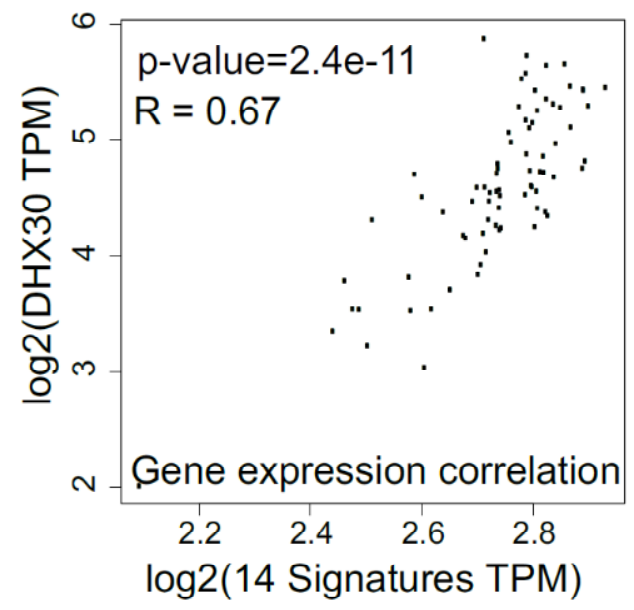

b

\section{LIHC}

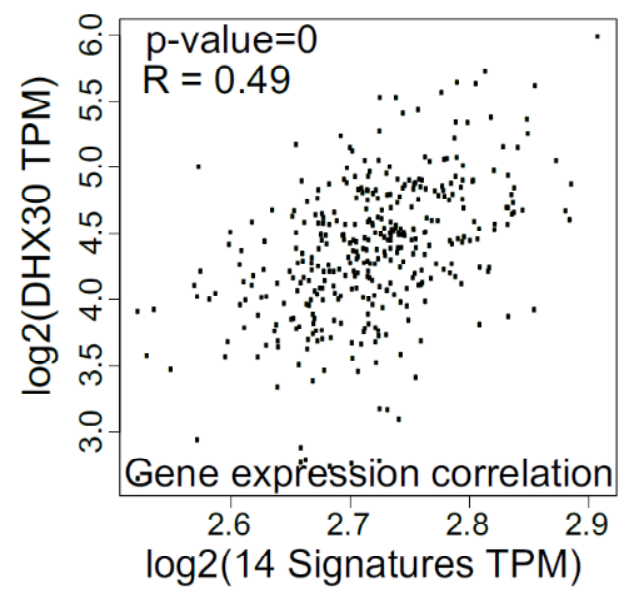

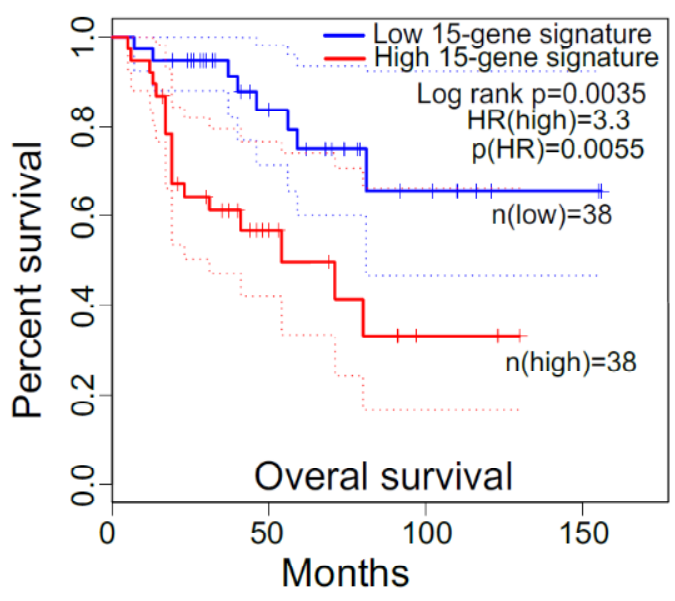

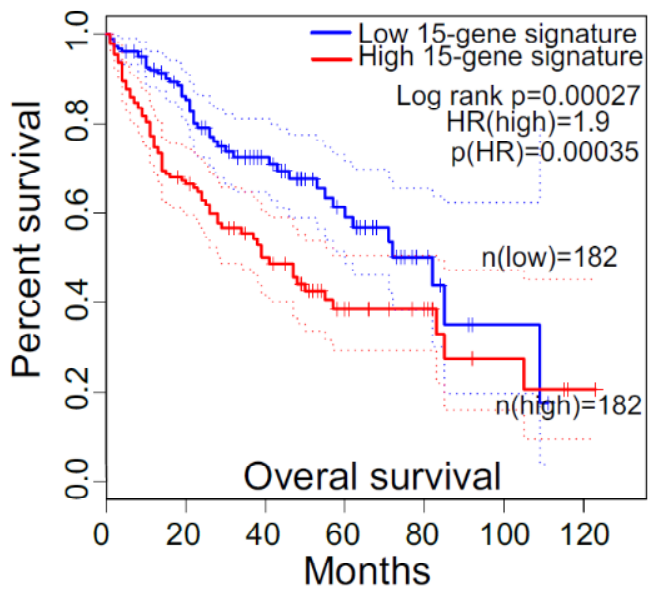

Figure 5. Prognostic value of a gene signature comprising DHX30 and its target mitoribosome transcripts. See also Figure S5.

TGCA data was interrogated through the GEPIA web resource (http://gepia2.cancer-pku.cn) to evaluate the correlation between the expression of DHX30 and that of 14 mitoribosomal transcripts that are predicted to be direct targets. Presented are two examples among a cluster of eleven cancer types where a positive correlation is apparent. See Figure S7 for the complete data and additional examples. A) Adrenocortical Carcinomas (ACC). B) Liver Hepatocellular Carcinomas (LIHC). Left panels, Pearson correlation; right panels, Overall Survival Kaplan-Meier. The correlation value, the Hazardous Ratio, p values and sample sizes are shown.

\section{Discussion}

Ribosome biogenesis and translation impose a high metabolic demand on the cell ${ }^{17,18}$. Hence, a coordination between translational control and metabolic output ultimately involving mitochondrial respiratory functions is expected to contribute to cell homeostasis and fitness ${ }^{19,20}$. However, relatively few proteins and pathways have been established to exert a direct role in balancing cytoplasmic translation initiation with mitochondrial metabolism ${ }^{21,22}$. eIF6 represents a significant example, as it has been clearly shown that it negatively controls $80 \mathrm{~S}$ monosome assembly, a necessary step for translation 
initiation, while at the same time playing a critical positive role in mitochondrial functions, as revealed by broad changes in the mitochondrial proteome in eIF6 hemizygous mice ${ }^{23-25}$.

We propose that DHX30 can also exert an important housekeeping role in coordinating ribosome biogenesis, translation, and mitochondrial respiration. DHX30 depletion in HCT116, stable or transient, leads to a modest but significant increase in ribosome biogenesis as well as in global translation; on the contrary, mito-ribosome transcripts, particularly of the large subunits, exhibit reduced translation efficiency. Furthermore, DHX30 can also exert a more direct role on mitochondria, as the protein can directly localize to the organelle, thanks to an alternative first exon that features a localization signal. The steady state expression of several mitochondrially encoded genes was reduced following the depletion of both DHX30 transcripts and perhaps more effectively, by the reduction of the mitochondrial isoform. A previous study provided clear evidence that DHX30 together with DDX28, FASTKD2, and FASTKD5 can promote the assembly of $55 \mathrm{~S}$ mito-monosome and translation ${ }^{4}$. Consistent with the relevance of a mitochondrial and translation function, the DHX30 transcript has been shown to be located and locally translated at the ER-Outer Mitochondrial membrane interface, by APEX-seq ${ }^{12}$. In fact, none of the DHX30 closer homologs showed strong evidence of such localized translation, or other evidence of mitochondrial localization.

Although our experiments did not enable us to confirm this in HCT116, a previous report also showed evidence for DHX30 interaction with mitochondrial transcripts in human fibroblasts by RIP-seq ${ }^{4}$. Our data instead point to a direct interaction with mitoribosome transcripts and their positive modulation as another means by which DHX30 can indirectly affect mitochondrial translation. We validated by RIP the binding of DHX30 to MRPL11 and MRPS22. Leveraging public data from eCLIP experiments in ENCODE and our polysomal profiling, RNA-seq, and GSEA analysis, we propose that DHX30 could directly interact with fourteen mito-ribosome protein transcripts. An even larger set of ribosomal protein transcripts are nominated as direct DHX30 targets by eCLIP ${ }^{9}$ and are showing changes in translation efficiency upon DHX30 depletion in HCT116 cells.

We observed that these two groupings of transcripts markedly differed for their basal translation efficiency, which was low for cytoplasmic ribosomal protein transcripts. Those mRNAs are known to contain particularly structured 5'-UTR and to be strongly regulated at the level of translation initiation by mTOR and MYC-regulated pathways ${ }^{26-28}$. The mechanism by which DHX30 can control ribosomal protein (RP) transcripts remains to be established. In a recent study, we discovered an RNA sequence motif in 3'UTRs, labeled CGPD, that is targeted by DHX30 and can mediate higher translation levels ${ }^{6}$. While we cannot exclude that the CGPD motif can be implicated, only a subset of RP transcripts harbors instances of it. Our de novo search for over-represented motifs did not retrieve another prominent candidate cis-element. An even lower number of mito-RP transcripts were found to harbor instances of the CGPD-motif. This was expected, as DHX30 is inferred to have an opposite role on RP and mito-RP transcripts based on the direction of changes in their TE observed in HCT116_shDHX30 cells. For the mito-RP gene group, a de novo motif over-representation search did not identify a strongly enriched ciselement.

The magnitude of fold change and translation efficiency changes for both ribosomal and mitoribosomal transcripts in response to DHX30 silencing is modest in absolute value. This could be in part due to a limitation of the experimental approach. As reported in our earlier study, a complete knockout of DHX30 does not seem to be attainable in HCT116 cells. Both our siRNA and even more so the shRNA clones retain partial DHX30 expression. Furthermore, it is important to emphasize that changes in the expression or even polysomal association of ribosomal protein transcripts do not necessarily predict translation rate changes. However, several endpoints consistently suggested to us that HCT116-shDHX30 cells exhibited higher ribosome number and increased global translation. When treated with an MDM2 inhibitor inducing strong p53-dependent transcriptional response, those cells were also shown to markedly alter their translatome ${ }^{6}$. While our results pointed to a direct role of DHX30 on translation specificity, we cannot exclude that the global effect on ribosome biogenesis we propose represents a 
constitutive, housekeeping function for DHX30 that would also contribute to the observed changes in translation specificity, under the notion that a change in global translation potential or in the modulation of translation initiation is not expected to impact equally every available transcript in a cell ${ }^{10,20}$.

Depletion of DHX30 reduced cell proliferation in various assays. This effect was visible also by transient silencing of just the cytoplasmic transcript, although it was more evident when both cytoplasmic and mitochondrial isoforms were targeted. Although we cannot exclude that the lower proliferation results from a checkpoint activation, as we have seen that in the siRNA experiments the lack of p53 can reduce the proliferation lag (Figure S8), cells did not show evidence for overt cell cycle arrest. This is not entirely unexpected, due to the residual levels of DHX30 expression in the cell models we used, as noted above, and also for the possible selective pressure for compensatory effects given the central nature of the processes involved. Transient silencing of DHX30 in HCT116 p53 null cells besides having a reduced impact on proliferation also did not significantly alter the expression of MRPS22, MRPL11 or of mitochondrial transcripts. While this may be suggestive of a functional interaction between DHX30 and

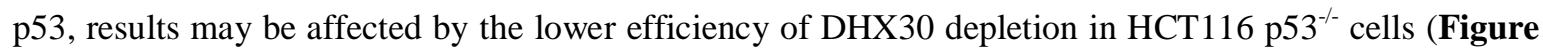
S8).

Mito-ribosomal proteins have been proposed to have additional, moonlighting functions unrelated to mito-ribosome biogenesis, in particular in the modulation of apoptosis. For example, MRPS29, also known as DAP3, was reported to influence the extrinsic apoptosis pathway ${ }^{29,30}$, while MRPL41 was proposed to modulate p53-dependent intrinsic apoptosis ${ }^{31}$. It is, however, unlikely that these potential pro-apoptotic functions can contribute to the proliferation defects seen after DHX30 depletion, given that their expression is not significantly modified. The expression of several mito-ribosome components has been evaluated as potential biomarkers associated with cancer clinical variables ${ }^{32}$.

Hence, we reasoned that changes in DHX30 levels to an extent that would not lead to overt stress responses could provide opportunities for increased fitness due to the coordination between mitochondrial function and translation potential, which could be considered as a balancing function between energy supply and demand. If any, the effects would be expected to reflect tissue-specific differences in metabolism. We explored TGCA data, first evaluating if a positive correlation could be observed in normal tissue and/or primary cancer samples for DHX30 and mito-ribosome gene expression. Crossreferencing eCLIP data, with our polysomal profiling, RNA-seq, RIP results, and GSEA data we compiled a list of 14 mito ribosomal transcripts as candidate direct DHX30 targets. Interestingly DHX30 and this 14 gene list showed a prognostic significance in cancer types where a positive correlation in their expression was observed. Instead, copy number alterations or mutations in DHX30 were a rare event in cancer $(8.6 \%)$, were not associated with specific tissue or cancer types, and, although based on a few cases, seemed to be mutually exclusive with mitochondrial genome changes (Figure S9).

In conclusion, our study demonstrated DHX30 role in the coordination between global cytoplasmic translation and mitochondrial functions contributes to the oncogenic potential of cancer cells, and appears to correlate with tumor aggressiveness and clinical outcome. Furthermore, during stress responses activating p53, DHX30 can reduce the apoptotic commitment of cancer cells by acting on specific pro-apoptotic transcripts, thus providing a potential actionable target for therapeutic purposes ${ }^{6}$.

\section{Materials and Methods}

\section{RNA-seq library preparation and sequencing}

shDHX30 and control cells were either kept untreated or treated with $10 \mu \mathrm{M}$ Nutlin, and processed after 12h. Polysomal profiling and RNA extraction of reconstituted total cytoplasmic fractions was performed as recently described ${ }^{6}$. Sequencing libraries were obtained following the manufacturer instructions of the TruSeq RNA Library preparation kit v2. We used $1.5 \mu \mathrm{g}$ of RNA as input, and assessed the input RNA 
quality by the Agilent RNA 6000 Nano kit on a Agilent 2100 Bioanalyzer instrument. Four replicates were generated for each condition. The resulting 16 samples were sequenced on a HiSeq 2500 machine producing $25 \mathrm{M}$ raw reads per each sample.

\section{RNA-seq data analysis}

Reads were first quality filtered and trimmed with trimmomatic (minimum quality 30 , minimum length 36nt) ${ }^{33}$. Then, each Gencode v27 (http://www.gencodegenes.org/releases/) transcript was quantified with Salmon ${ }^{34}$. Eventually, edgeR ${ }^{35}$ was used to call Differentially Expressed Genes (DEGs) between conditions at the polysomal and total levels (shDHX30 DMSO vs shNT DMSO), using a 0.05 threshold on the adjusted p-value. GSEA was performed with the fgsea $\mathrm{R}$ package ${ }^{8}$, including the Hallmark, Canonical Pathways, and GO gene sets. We used 1000 permutations to compute the significance p-value and used a BH adjusted p-value threshold of 0.05. Translational efficiency (TE) was computed on normalized expression data (counts per million reads, CPM) as the ratio of polysomal CPMs over total CPMs for each replicate and transcript in the annotation. Differences in TE (between conditions and groups of genes) and between polysomal and total samples were assessed by the Wilcoxon test. Motifs in ribosomal and mitoribosomal genes 5'- and 3'-UTRs were obtained with DREME ${ }^{36}$ using a 1.0E-04 pvalue threshold and shuffled input sequences as controls.

\section{Cell lines and culture conditions}

HCT116, U2OS and MCF-7 shNT control or shDHX30 clones were obtained as recently described ${ }^{6}$. HCT116 p53 ${ }^{-/-}$cells were obtained from the Vogelstein lab (Sur S. et al, 2009). All cell lines were cultured in RPMI (Gibco, Thermo Fisher Scientific, Waltham, MA, USA) supplemented with 10\% Fetal Bovine Serum (Gibco, Thermo Fisher Scientific), 1X L-Glutamine and Pen/Strep (Gibco, Thermo Fisher Scientific) at $37{ }^{\circ} \mathrm{C}$ with $5 \% \mathrm{CO} 2$ in a humidified atmosphere. U2OS cells were cultured in DMEM pH 7.4 (Gibco, Thermo Fisher Scientific) supplemented with 10\% Fetal Bovine Serum (Gibco, Thermo Fisher Scientific), 1X L-Glutamine and Pen/Strep (Gibco, Thermo Fisher Scientific) at $37{ }^{\circ} \mathrm{C}$ with $5 \%$ $\mathrm{CO} 2$ in a humidified atmosphere. For routine culture of depleted clones and controls, $0.2 \mu \mathrm{g} / \mathrm{mL}$ Puromycin was added to the culture medium to maintain selection of the vectors. Puromycin was removed 24 hours before starting a specific experiment to avoid confounding effects. MCF-7, U2OS and HCT116 parental and $\mathrm{p} 53^{-/-}$cells were silenced for cytoplasmic or cytoplasmic + mitochondrial DHX30 variants using $25 \mathrm{nM}$ siRNAs (Trifecta, IDT, Coralville, IA, USA) (Table S2) transfected with Interferin (Polyplus-transfection, Illkirch, France). All experiments were performed at least 24 hours post-silencing.

\section{Western Blot}

Cells were cultured in 6-well tissue culture plates. Cells were collected with trypsin-EDTA after 24 hours, centrifuged and washed with PBS. Samples were then lysed with RIPA buffer () and the proteins were quantified by BCA assay (EUROCLONE, Milan, Italy). $30 \mu \mathrm{g}$ of extracted proteins were loaded on $12 \%$ or 15\% Tris-glycine Gel and then transferred onto a nitrocellulose membrane using Tris-Glycine buffer. Blocking was performed overnight with 5\% not-fat dry milk, 0.1\% TWEEN and PBS 1X. Immunodetection was obtained using primary and secondary antibodies reported in Table S3. Membranes were analyzed by ECL and detected with ChemiDocTM XRS+ (Bio-Rad, Hercules, CA, USA) using ImageLab software (Bio-Rad).

\section{Polysome profiling}

Polyribosome analysis was performed as described in ${ }^{37-39}$. Briefly, HCT116 cells were grown on $15 \mathrm{~cm}$ Petri dishes with standard media and serum. When the cells reached $80 \%$ of confluence, cycloheximide $(0.01 \mathrm{mg} / \mathrm{ml})$ was added and kept in incubation for $10 \mathrm{~min}$. Then cells were washed two times with cold 
PBS containing cycloheximide $(0.01 \mathrm{mg} / \mathrm{ml})$ and lysed with the following lysis buffer: $20 \mathrm{mM}$ Tris-HCl (pH 7.5), $100 \mathrm{mM} \mathrm{KCl}, 5 \mathrm{mM} \mathrm{MgCl} 2,0.5 \%$ Nonidet P-40, $100 \mathrm{U} / \mathrm{ml}$ RNase inhibitors. Mitochondria and nuclei-free lysates were loaded onto $15-50 \%(\mathrm{w} / \mathrm{v})$ density sucrose gradients in salt solution (100 mM $\mathrm{NaCl}, 5 \mathrm{mM} \mathrm{MgCl} 2,20 \mathrm{mM}$ Tris- $\mathrm{HCl} \mathrm{pH}$ 7.5), and ultracentrifuged at $180000 \times \mathrm{g}$ for $100 \mathrm{~min}$ at $4^{\circ} \mathrm{C}$. The sedimentation profiles were monitored by absorbance at $254 \mathrm{~nm}$ using a Teledyne ISCO UA- 6 fractionator coupled to UV detector, collecting thirteen $1 \mathrm{ml}$ fractions. RNA from pooled polysomal fraction was extracted and processed as described in (R Z 2020). For Western blot analysis, $100 \mathrm{ul}$ of $100 \%$ TCA and $1 \mathrm{ml}$ of ice-cold acetone were added to $1 \mathrm{ml}$ of each fraction. Then samples were put at $-80{ }^{\circ} \mathrm{C}$ overnight to induce protein precipitation. Subsequently samples were centrifuged at $16,000 \times \mathrm{g}$ for $10 \mathrm{~min}$ at $4{ }^{\circ} \mathrm{C}$ and washed three times with $1 \mathrm{~mL}$ of ice-cold acetone. Finally, the pellets were solubilized directly in Laemmli buffer $\mathrm{pH} 8$.

\section{Ribosome isolation}

Ribosome isolation was performed following the protocol described by ${ }^{40}$. Briefly, $8 \times 10^{6}$ cells were seeded in a T150 flask, 48 hours before the procedure. At $80 \%$ of confluence, cells were detached and counted. $1 \times 10^{7}$ cells were pelleted by $500 \mathrm{x}$ g centrifugation for 5 minutes at $4^{\circ} \mathrm{C}$ and washed once with cold PBS. Supernatant was discarded and the pellet was re-suspended gently with $300 \mu \mathrm{L}$ of cold buffer A

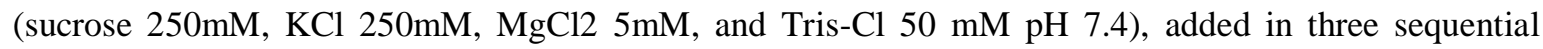
steps, pipetting after every addition. To perform cell lysis, an appropriate volume of NP-40 was added to the homogenized cellular solution, in order to obtain a $0.7 \%(\mathrm{v} / \mathrm{v})$ final concentration of the detergent, and cells were incubated on ice for 15 minutes, homogenizing the suspension by gentle pipetting every 5 minutes. Then the cell lysate was centrifuged at $750 \mathrm{x}$ g for 10 minutes at $4^{\circ} \mathrm{C}$ to pellet nuclei; the recovered cytoplasmic fraction (supernatant) was centrifuged again at $12.500 \mathrm{x} \mathrm{g}$ for 10 minutes at $4^{\circ} \mathrm{C}$ to obtain a mitochondria pellet. Supernatant containing ribosomes was collected and its volume was accurately measured using a graduated pipet. $\mathrm{KCl} 4 \mathrm{M}$ solution was then slowly added in order to reach a final concentration of $0.5 \mathrm{M}$. Meanwhile, $1 \mathrm{~mL}$ of the sucrose cushion solution (sucrose $1 \mathrm{M}, \mathrm{KCl} 0.5 \mathrm{M}$, $\mathrm{MgCl} 25 \mathrm{mM}$ and Tris- $\mathrm{Cl} 50 \mathrm{mM} \mathrm{pH} 7.4$ ) was added into 3-mL polycarbonate tube for an ultracentrifuge TL100.3 rotor (Beckman Coulter, Brea, CA, USA). The KCl-adjusted ribosome-containing solution was carefully added above the sucrose cushion and tubes were balanced by weight using buffer $\mathrm{B}$ (sucrose $250 \mathrm{mM}, \mathrm{KCl} 0.5 \mathrm{M}, \mathrm{MgCl} 25 \mathrm{mM}$ and Tris-Cl $50 \mathrm{mM} \mathrm{pH} \mathrm{7.4).} \mathrm{Then,} \mathrm{they} \mathrm{were} \mathrm{ultracentrifuged} \mathrm{at}$ $250.000 \mathrm{x}$ g for 2 hours at $4^{\circ} \mathrm{C}$. At the end of the centrifugation, the supernatant was discarded and a very compact and dense translucent pellet containing ribosomes was quickly rinsed twice by carefully adding $200 \mu \mathrm{L}$ of cold water. Then, pellet was re-suspended in $300 \mu \mathrm{L}$ of buffer $\mathrm{C}(\mathrm{KCl} 25 \mathrm{mM}, \mathrm{MgCl} 25 \mathrm{mM}$ and Tris-Cl 50mM pH 7.4), adding the solution in three sequential steps and gently homogenized by pipetting after every $100 \mu \mathrm{L}$ addition. Finally, to estimate the amounts of ribosomes, absorbance at $260 \mathrm{~nm}$ of the suspensions was measured by a nanodrop spectrophotometer (ThermoFisher).

\section{Global Translation}

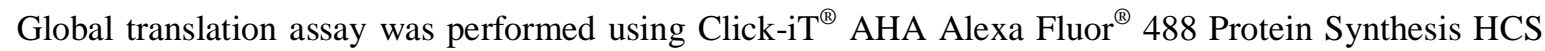
Assay (Thermo Fisher Scientific). Briefly, HCT116 shNT and shDHX30 cells were cultured in 96-well tissue culture plate and treated with 100mM 5-Fluorouracil (Sigma Aldrich) as positive control. After 24 hours of treatment, the culture medium was removed and $50 \mu \mathrm{M}$ L-azidohomoalanine prepared in prewarmed L-methionine-free medium (Lonza) was added and cells were incubated for 1 hour. Cell fixation was performed using 3.7\% formaldehyde (Sigma Aldrich) incubating the plate at room temperature for 15 minutes. After two washing with 3\% BSA in PBS $1 \mathrm{X}$ cells were permeabilized with $0.5 \%$ Triton ${ }^{\circledR} \mathrm{X}-100$ (Sigma Aldrich) and incubated for 15 minutes at room temperature. Cells were washed twice with 3\% BSA in PBS $1 \mathrm{X}$ and Click-iT ${ }^{\circledR}$ reaction cocktail was added incubating for 30 minutes at room temperature, protected from light. After the incubation reaction cocktail was removed, cells were washed twice with 3\% BSA in PBS $1 \mathrm{X}$ and stained with $5 \mu \mathrm{g} / \mathrm{mL}$ Hoechst (Sigma Aldrich). Samples' images 
were acquired and analysed by high content fluorescence microscope Operetta ${ }^{\circledR}$ (PerkinElmer, Waltham, MA, USA) using the following filter set: Alexa Fluor ${ }^{\circledR}$ 488: Ex495/Em519 nm; Hoechst: Ex350/Em461 $\mathrm{nm}$.

\section{Fluorescent In Situ Hybridization (FISH) on rRNA precursors}

Cells were cultured on a coverslip place onto a 48 -well plate and after 48 hours they were washed with PBS and fixed with 4\% Paraformaldehyde (PFA) (Sigma Aldrich) for 30 minutes at RT. Then, cells were washed twice with PBS $1 \mathrm{X}$ and incubated in $70 \%$ ethanol overnight at $4{ }^{\circ} \mathrm{C}$. The day after, cells were rehydrated with $10 \%$ formamide in 2X SSC (Sigma Aldrich), twice for 5 minutes at RT. Meanwhile, buffer A [ $5 \mu \mathrm{L}$ formamide, $2.5 \mu \mathrm{L}$ SSC $2 \mathrm{X}, 2.5 \mu \mathrm{L}$ tRNA $(10 \mathrm{ng} / \mu \mathrm{L})$, water up to $20.25 \mu \mathrm{L}$ and then $2.5 \mu \mathrm{L}$ of each probe $(10 \mathrm{ng} / \mu \mathrm{L})]$ was incubated for 5 minutes at $90^{\circ} \mathrm{C}$ and then mixed quickly together with

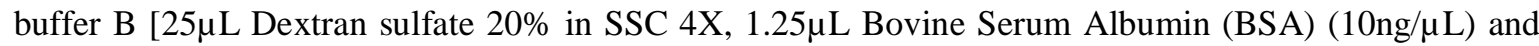
$2.5 \mu \mathrm{L}$ ribonucleoside vanadyl complex (RVC) $(200 \mathrm{mM})]$. Then, $50 \mu \mathrm{L}$ A+B probe solution (Table S2) was dropped onto the coverslip and it was incubated in a humidified chamber for 3 hours at $37^{\circ} \mathrm{C}$. When the incubation was finished, the coverslip was washed twice with $10 \%$ formamide in SSC $2 \mathrm{X}$ for 30 minutes at RT and once with PBS $1 \mathrm{X}$ for 5 minutes. Cells were stained with a solution of $0.5 \mu \mathrm{g} / \mathrm{mL}$ DAPI (Sigma Aldrich) in PBS 1X for 5 minutes, washed three times with PBS 1X and the coverslip was finally placed with mounting medium on glass slide. Finally, images were acquired using Zeiss Observer z1 fluorescent microscope (Carl Zeiss, Oberokochen, Germany) with ZEN 2 blue edition software ver. 2.3 (Carl Zeiss). Images' fluorescence intensity was measured using Cell Profiler software ver. 3.1.9 (Broad Institute, Cambridge, MA, USA).

\section{rRNA biogenesis}

rRNA biogenesis was performed following the protocol described by ${ }^{41,42}$. Briefly, cells were cultured in 96-well tissue culture plates and after 24 hours were treated with Actinomycin D 100nM (Sigma Aldrich) as positive control. After 24 hours of treatment, $1 \mathrm{mM}$ 5-Ethynyluridine (Sigma Aldrich) was added to the culture medium and the plate was incubated for 2 hours. This short incubation with 5-EU allows a higher rate of incorporation in rRNAs than mRNAs, reducing the background signal. Then, cells were fixed using a working solution 1 (WR1) composed by $125 \mathrm{mM}$ Pipes pH 6.8 (Sigma Aldrich), 10mM EGTA (Sigma Aldrich), 1mM MgCl2 (Merck Millipore, Burlington, MA, USA), 0.2\% Triton ${ }^{\circledR}$ X-100 (Sigma Aldrich) and 3.7\% formaldehyde (Sigma Aldrich). Cells were washed twice with TBS $1 \mathrm{X}$ and stained for 30 minutes at room temperature using a working solution 2 (WR2) composed by $100 \mathrm{mM}$ Tris- $\mathrm{HCl} \mathrm{pH} 8.5$ (Sigma Aldrich), 1mM CuSO4 (Merck Millipore), 10 $\mu \mathrm{M}$ fluorescent azide (Sigma Aldrich) and 100mM ascorbic acid (Merck Millipore). After four washes with TBS containing 0.5\% Triton X-100, cells were stained with $0.5 \mu \mathrm{g} / \mathrm{mL}$ Hoechst (Sigma Aldrich) and samples' images were acquired and analysed by high content fluorescent microscope Operetta ${ }^{\circledR}$ (PerkinElmer) using the following filter set: Alexa Fluor ${ }^{\circledR}$ 488: Ex495/Em519 nm; Hoechst: Ex350/Em461 nm and setting a threshold on Alexa Fluor® 488 at 500 fluorescent units to plot the percentage of cells with high nucleolar signal intensity.

\section{Colony formation}

1.0x10 $0^{3}$ HCT116 shNT and shDHX30 cells/well were seeded in a 6-well plate and incubated. Culture medium was changed every three days. Colonies were ex fixed with $3.7 \%$ formaldehyde and stained with a solution containing 30\% Methylene blue (Sigma Aldrich) in water, then washed five times for 5 minutes. Images were acquired and analysed using ImageJ software 1.8.0 (National Institute of Health, Bethesda, MD, USA).

\section{Cell Count in high content analysis and Real Time Cell Index Analysis}

To analyze cell proliferation by cell count, $1.0 \times 10^{3}$ cells/well were seeded in 96-well plates and incubated. Images were acquired by high content fluorescent microscope Operetta ${ }^{\circledR}$ (PerkinElmer) in 
digital phase contrast for three days. To analyse cell proliferation by Real Time Cell Analyser xCELLigence $^{\circledR}$ (RTCA) (Roche, Basel, Switzerland) the background was set adding the only cell culture medium in each well of E-plate and incubating 30 minutes. After incubation, background was read. Then $1.0 \times 10^{3}$ cells/well were seeded and incubated for 30 minutes and then the E-plate was inserted in the RTCA and impedance-based cell proliferation was estimated by RTCA readings every 15 minutes in the course of three days.

\section{Spheroid assay formation}

$3.0 \times 10^{3}$ HCT116 shNT and shDHX30 cells/well were seeded in U-bottom ultra-low attachment 96-well plate (Corning Incorporated, Corning, NY, USA), centrifuged at $3000 \mathrm{rpm}$ for 5 minutes and incubated. For MCF-7, $100 \mu \mathrm{L}$ of cold medium containing $3.0 \times 10^{3}$ cells/well were mixed with $2.5 \%$ of Cultrex® $\square$ BME (Trevigen, Gaithesburg, MD, USA), centrifuged at $5000 \mathrm{rpm}$ for 10 minutes at $4^{\circ} \mathrm{C}$ and incubated. Starting after three days of incubation, images were acquired every 24 hours for eight additional days by microscope (Leica, Wetzlar, Germany). Spheroids' area was measured by ImageJ software.

\section{Immunofluorescence}

3.0x105 cells/well were seeded on glass coverslip inserted in 6-well plates and incubated overnight. The days after, cells were fixed in $4 \%$ Paraformaldehyde (PFA) for $15 \mathrm{~min}$. Then the PFA solution was removed and cells were rinsed twice with PBS 1X. After washing, cells were incubated for 1 hour with blocking solution (PBS 1X, 5\% BSA and 0.5\% Triton X-100). Next, cells were incubated overnight at $4^{\circ} \mathrm{C}$ with primary antibodies (Table 2.4) diluted in blocking solution. The following day, after three washes with PBS 1X, the anti-mouse and anti-rabbit secondary antibodies conjugated with AlexaFluor 488 or AlexaFluor 594 (Invitrogen, Carlsbad, CA, USA) (diluted 1:1000) were added to the samples and incubated for 1 hour at RT under agitation. Cells were washed with PBS 1X three times and cell nuclei were stained with $0.5 \mu \mathrm{g} / \mathrm{mL}$ Hoechst (Sigma Aldrich) (diluted 1:5000). Finally, coverslips were mounted on glass slides and images were acquired using Zeiss Observer Z1 fluorescent microscope and Zen $2012 \AA$ software (Carl Zaiss Sport Optics, Wetzlar, Germany). The list of primary antibodies and dilutions used are reported in Table $\mathbf{S 3}$.

\section{Compensatory Glycolytic Test}

3.0x $10^{3}$ cells/well were seeded in Xfp cell culture microplate (Agilent Technologies, Santa Clara, CA, USA) and incubated overnight while XF calibrant solution was added in each well of extracellular flux cartridge and incubated overnight without $\mathrm{CO}_{2}$. The day after, culture media was removed from cells, replaced with assay medium and microplate was incubated 45 minutes at $37^{\circ} \mathrm{C}$ without $\mathrm{CO}_{2}$. Extracellular flux cartridge was prepared adding $0.5 \mu \mathrm{M}$ rotenone/antimycin $\mathrm{A}$ in port $\mathrm{A}$ and $50 \mathrm{mM}$ 2-Deoxyglucose in port B diluted in Xfp assay medium and the cartridge was calibrated by Seahorse XFp analyser setting Glycolysis Test program. When the calibration was finished, cell microplate was inserted in a Seahorse XFp analyser starting the analysis.

\section{RNA extraction and qRT-PCR}

$3.0 \times 10^{5}$ cells/well were seeded in a 6-well plate and incubated overnight. The day after, cells were starved and lysed with $\beta$-mercaptoethanol and RNA was extracted using Illustra RNAspin Mini RNA Isolation kit (GE-Healthcare, Chicago, IL, USA) following the kit protocol. Then, RNA was retro-transcribed using the RevertAid RT Kit (Thermo Fisher Scientific). Finally, RT-qPCR was performed using 25ng and 2x qPCR SyGreen (PCR Biosystem, London, England) in Cfx96 ${ }^{\mathrm{TM}}$ Real-Time System or Cfx384 ${ }^{\mathrm{TM}}$ RealTime System Thermocyclers (Bio-Rad). The sequence of all primers used is reported in Table S2.

\section{Digital droplet PCR}


Digital droplet PCR protocol used was based on that developed by Bio-Rad. Briefly, $1 \times 10^{5}$ cells were seeded in 6-well plate and grown for 1 day. Next cells were detached, counted and solubilized in DireCtQuant 100ST solubilization reagent (DireCtQuant, Lleida, Spain) at the concentration of 1000 cells $/ \mu \mathrm{L}$, incubating for 3 minutes at $90^{\circ} \mathrm{C}$ with shaking at $750 \mathrm{rpm}$ and then centrifuged for 1 minute at $10000 \mathrm{~g}$. Lysates were obtained by incubation for 3 minutes at $90^{\circ} \mathrm{C}$ under shaking at $750 \mathrm{rpm}$ and then centrifuged for 1 minute at $10000 \mathrm{~g}$. Lysate was diluted 1:4 in solubilization reagent and for every target to analyze, PCR mix was prepared comprising $2 \mu \mathrm{L}$ of diluted sample, forward and reverse primers at $10 \mu \mathrm{M}$ final concentration in a final volume of $95 \mu \mathrm{L}$. PCR mix without lysate was used as negative control. Then, $10.5 \mu \mathrm{L}$ of each PCR reaction was mixed with $11 \mu \mathrm{L}$ of $2 \times \mathrm{QX} 200$ ddPCR EvaGreen SuperMix (Bio-Rad) and $0.5 \mu \mathrm{L}$ of HindIII restriction enzyme (NEB, Ipswich, MA, USA). Sample DNA was digested at $37^{\circ} \mathrm{C}$ for 15 minutes and loaded in ddPCR DG8 ${ }^{\mathrm{TM}}$ Cartridge with QX200 ${ }^{\mathrm{TM}}$ droplet generation oil (Bio-Rad). Droplets were made using QX200 ${ }^{\mathrm{TM}}$ droplet generator (Bio-Rad) and then loaded in a 96-well PCR plate. Sample DNA was amplified and finally, droplets with amplified DNA were analyzed using the QX200"T droplet reader and QuantaSoft 1.7.4 (Bio-Rad).

\section{RNA Immunoprecipitation}

HCT116 cells were cultured in standard medium in P150 plates till they reached $\sim 80 \%$ confluence. $\sim 10^{7}$ cells were lysed in $1 \mathrm{~mL}$ of Lysis buffer $(100 \mathrm{mM} \mathrm{KCl}, 5 \mathrm{mM} \mathrm{MgCl} 2,10 \mathrm{mM}$ HEPES pH 7, $0.5 \%$ NP-40, $1 \mathrm{mM}$ DTT, 1U/ul RNase Inhibitors, 1X Protease Inhibitor Cocktail) using a scraper. Lysates were transferred in a falcon tube, placed for at least two hours at $-80^{\circ} \mathrm{C}$, and centrifuged at $10000 \mathrm{rpm}$ for 30 minutes. Supernatants were collected in a new tube. Dynabeads ProteinA or ProteinG (depending on the antibody species, Thermo Fisher scientific) were prepared by washing them twice with NT2 Buffer (50mM Tris- $\mathrm{HCl} \mathrm{pH} 7.4,150 \mathrm{mM} \mathrm{NaCl}, 1 \mathrm{mM} \mathrm{MgCl} 2 ; 0.05 \%$ NP40) and resuspended in NT2 buffer. Beads were distributed in different tubes, supplemented with twice their initial volume of NT2 Buffer. The DHX30 specific antibody (5 $\mu \mathrm{g}$-A302-218A, Bethyl) or IgGs were added to the beads and incubated for 2 hours on a rotating wheel at $4^{\circ} \mathrm{C}$. Lysates were pre-cleared by adding a mix containing Protein $\mathrm{A}$ and ProteinG Dynabeads in equal amounts and incubating for 1 hour at $4{ }^{\circ} \mathrm{C}$ on a wheel. After placing the tubes on a magnet, supernatants were collected and $1 \%$ of their volume was used as input to be directly extracted with TRIzol. The remaining supernatant was added to the antibody-coated beads and incubated overnight on a wheel at $4^{\circ} \mathrm{C}$. The day after, beads were resuspended in $1 \mathrm{ml} \mathrm{NT2}$ buffer, transferred in a new tube and washed with $1 \mathrm{ml}$ NT2 buffer for 10 minutes on a wheel at $4^{\circ} \mathrm{C}$. All the washes were performed on a magnet. Three additional washes were performed again with $1 \mathrm{ml}$ NT2 Buffer supplemented with $0.1 \%$ Urea $+50 \mathrm{mM} \mathrm{NaCl}\left(10\right.$ minutes each at $4{ }^{\circ} \mathrm{C}$ on a wheel). Beads were washed one more time in $500 \mu 1$ of NT2 buffer, $50 \mu 1$ were collected for WB analysis and the remaining supernatant was discarded. RNA was extracted by adding TRIzol to the beads, according to the manufacturer's protocol. The RNA pellets were resuspended in $15 \mu$ l DEPC water and cDNAs were synthesized using the RevertAid First Strand cDNA Synthesis Kit (Thermo Fisher Scientific).

\section{Cytoplasm-Mitochondria fractionation}

For cytoplasm-mitochondria fractionation, $2 \times 10^{6}$ cells were seeded in P150 Petri dish and incubated for 2 day. Then, they were detached using trypsin and re-suspended in $750 \mu \mathrm{L}$ of Mitochondrial Isolation Buffer (MIB) (0.32M Sucrose, 1mM EGTA pH 8.0, 20mM Tris-HCl pH 7.2) with $0.1 \%$ fatty acid-free BSA. Cells were homogenized in a Potter-Elvehjem homogenizer (DWK Life Sciences, Mainz, Germany) applying more than 60 strokes. Homogenate (A) was centrifuged for 5 minutes at $1000 \mathrm{~g}$ at $4{ }^{\circ} \mathrm{C}$ and supernatant was collected. Cell debris were re-suspended in $750 \mu \mathrm{L}$ of MIB + BSA and re-homogenized with more than 20 strokes. New homogenate (B) was centrifuged for 5 minutes at $1000 \mathrm{~g}$ at $4{ }^{\circ} \mathrm{C}$ and the supernatant was collected and pooled with supernatant $\mathrm{A}$. Two mixed supernatants $(\mathrm{A}+\mathrm{B})$ were centrifuged at $12000 \mathrm{~g}$ for 10 minutes at $4^{\circ} \mathrm{C}$ to pellet the mitochondria. Supernatant was discarded and mitochondria were washed twice with $1 \mathrm{~mL}$ of MIB + BSA and once with $1 \mathrm{~mL}$ of MIB, centrifuging the 
mitochondrial pellet every time at $12000 \mathrm{~g}$ for 10 minutes at $4^{\circ} \mathrm{C}$. Finally, supernatant was discarded and mitochondria were lysed in RIPA buffer and mitochondrial proteins were quantified by BCA assay.

\section{Apoptosis assay}

Apoptosis analysis was based on FITC Annexin-V Apoptosis Detection Kit protocol (BD Biosciences, San Jose, CA, USA). Briefly, $3 \times 10^{5}$ cells/well were seeded in a 6 -well plate, incubated for one day and then treated with drugs under investigation for 48 hours. 0.1\% DMSO was used as negative control. Cells were detached using trypsin and washed twice with PBS $1 \mathrm{X}$. Then, $1.5 \times 10^{5}$ cells were resuspended in $100 \mu \mathrm{L}$ of Binding Buffer 1X, stained with FITC-Annexin V and PI and incubated for 15 minutes at room temperature in the dark. Finally, samples were analyzed by FACSCanto ${ }^{\mathrm{TM}}$ flow cytometer and BD Diva Software 6.1.3. Unstained, PI-only and FITC-only stained samples were used to set the cytometer's parameters.

\section{Cell cycle analysis}

HCT shNT or shDHX30 were seeded in 6-well plates at a concentration of $3 \times 10^{5}$ cell/well. 48 hours after seeding, cells were trypsinized, washed with $1 \mathrm{X}$ PBS and counted for subsequent staining according to BD Cycletest ${ }^{\mathrm{TM}}$ Plus DNA Kit protocol. Briefly, $5 \times 10^{5}$ cells were centrifuged, superanatnt was removed and $250 \mu \mathrm{l}$ of Solution A were added. Cells were resuspended and incubated for 10 minutes before adding $200 \mu \mathrm{l}$ of Solution B. After 10 minutes of incubation, $200 \mu \mathrm{l}$ of ice-cold Solution C were added to the samples and cells were incubated for 10 minutes at $4^{\circ} \mathrm{C}$ in the dark before analysis with FACS CantoA (BD). The percentage of cells in each stage of the cell cycle (G1, S or G2) was computed using the ModFIT LT 4.0 software (Verity Software House).

\section{Exploration of TGCA data using the GEPIA web resource}

The GEPIA API ${ }^{15,16}$ was used to extract correlations between DHX30 and mitoribosomal genes (either as individual genes or for the 14-genes signature) in all available TCGA tumor datasets (correlation mode). The same analysis was performed to correlate cytoplasmic ribosome and mitochondrial ribosome genes expression, on TCGA tumor samples, matched TCGA normal samples, and GTEX healthy tissues RNAseq data. Survival analysis was performed with the same tool and in the same datasets, using the survival mode. Only results within the $\mathrm{p}$-value threshold of 0.05 were considered, with the others being set to $\mathrm{R}=0$ and $\mathrm{HR}=1$ in the heatmap for visualization purposes.

\section{Statistical analysis}

Data are presented as mean \pm SD of three independent biological experiments, unless stated otherwise. GraphPad Prism 5 or 8 software (GraphPad software, La Jolla, CA, USA) were used and either a two-way anova with Tukey's multiple comparison test or a two-tailed Student's t-test was performed, unless specified otherwise. Statistical significance was indicated as: ${ }^{*} \mathrm{p}<0.05 ; * \mathrm{*} p<0.01$; *** $<0.001$.

\section{Data availability}

The RNA-seq datasets are available in GEO with ID GSE95024 and GSE154065.

\section{Acknowledgements}

We thank Pamela Gatto, Viktoryia Sirarovich and Michael Pancher of the HTS CIBIO core facility for assistance with droplet digital PCR experiments, high-content as well as impedance-based proliferation assays. We thank Nicoletta di Bernardo for technical assistance for the apoptosis assays. This work was supported by the Italian Association for Cancer Research (Fondazione AIRC, IG grant \#18985 to AI). JJD laboratory is supported by grants by the Institut National du Cancer (INCa, grant PRT-K program 
$\mathrm{n}^{\circ}$ 2018-024 EMT-CoNCEPT) and Agence Nationale de la Recherche (ANR, grant RiboCard ANR-18CE11-0020, and grant ActiMeth ANR-19-CE12-0004).

\section{Conflict of interest}

The Authors declare no competing interest.

\section{Authors contributions}

B.B. perfomed most experiments and wrote the first draft of the paper. A.R., D.R., S.G., A.P., F.B., and, A.G. helped in some experimental procedures. F.C. and J-J.D. helped to design some experiments. E.D. performed all data analysis. E.D. and A.I. designed the study. A.I. co-wrote the first draft of the manuscript.

\section{References}

1 Zheng HJ, Tsukahara M, Liu E, Ye L, Xiong H, Noguchi S et al. The novel helicase helG (DHX30) is expressed during gastrulation in mice and has a structure similar to a human DExH box helicase. Stem Cells Dev 2015; 24: 372-383.

2 Ye P, Liu S, Zhu Y, Chen G, Gao G. DEXH-Box protein DHX30 is required for optimal function of the zinc-finger antiviral protein. Protein Cell 2010. doi:10.1007/s13238-010-0117-8.

3 Lessel D, Schob C, Kury S, Reijnders MRF, Harel T, Eldomery MK et al. De Novo Missense Mutations in DHX30 Impair Global Translation and Cause a Neurodevelopmental Disorder. Am J Hum Genet 2017; 101: 716-724.

4 Antonicka H, Shoubridge EA. Mitochondrial RNA Granules Are Centers for Posttranscriptional RNA Processing and Ribosome Biogenesis. Cell Rep 2015; 10: 920-932.

5 Andrysik Z, Galbraith MD, Guarnieri AL, Zaccara S, Sullivan KD, Pandey A et al. Identification of a core TP53 transcriptional program with highly distributed tumor suppressive activity. Genome Res 2017; 27. doi:10.1101/gr.220533.117.

6 Rizzotto D, Zaccara S, Rossi A, Galbraith MD., Andrysik Z, Pandey A et al. Nutlin-Induced Apoptosis is Specified by a Translation Program Regulated by PCBP2 and DHX30. Cell Rep 2020; 30: 1-15.

7 Tovar C, Rosinski J, Filipovic Z, Higgins B, Kolinsky K, Hilton H et al. Small-molecule MDM2 antagonists reveal aberrant p53 signaling in cancer: implications for therapy. Proc Natl Acad Sci U S A 2006; 103: 1888-1893.

8 Sergushichev AA. An algorithm for fast preranked gene set enrichment analysis using cumulative statistic calculation. bioRxiv 2016. doi:10.1101/060012.

9 Van Nostrand EL, Pratt GA, Shishkin AA, Gelboin-Burkhart C, Fang MY, Sundararaman B et al. Robust transcriptome-wide discovery of RNA-binding protein binding sites with enhanced CLIP (eCLIP). Nat Methods 2016; 13: 508-514.

10 Mills EW, Green R. Ribosomopathies: There's strength in numbers. Science (80-. ). 2017; : eaan2755.

11 Wang Y, Bogenhagen DF. Human mitochondrial DNA nucleoids are linked to protein folding machinery and metabolic enzymes at the mitochondrial inner membrane. J Biol Chem 2006; 281: 25791-802.

12 Fazal FM, Han S, Parker KR, Kaewsapsak P, Xu J, Boettiger AN et al. Atlas of Subcellular RNA Localization Revealed by APEX-Seq. Cell 2019. doi:10.1016/j.cell.2019.05.027.

13 Heiden MGV, Cantley LC, Thompson CB. Understanding the warburg effect: The metabolic requirements of cell proliferation. Science (80-. ). 2009; : 324.

14 Schwanhüusser B, Busse D, Li N, Dittmar G, Schuchhardt J, Wolf J et al. Global quantification of mammalian gene expression control. Nature 2011; 473: 337-342.

15 Tang Z, Li C, Kang B, Gao G, Li C, Zhang Z. GEPIA: A web server for cancer and normal gene expression profiling and interactive analyses. Nucleic Acids Res 2017; 45: W98-W102.

16 Tang Z, Kang B, Li C, Chen T, Zhang Z. GEPIA2: an enhanced web server for large-scale expression profiling and interactive analysis. Nucleic Acids Res 2019; 47: W556-W560.

17 Wieser W, Krumschnabel G. Hierarchies of ATP-consuming processes: Direct compared with 
indirect measurements, and comparative aspects. Biochem J 2001; : 389-95.

J 1995; : 163-7.

19 Couvillion MT, Soto IC, Shipkovenska G, Churchman LS. Synchronized mitochondrial and cytosolic translation programs. Nature 2016; 533: 499-503.

20 Calamita P, Gatti G, Miluzio A, Scagliola A, Biffo S. Translating the game: Ribosomes as active players. Front Genet 2018; 15: 533.

$21 \quad$ Pla $\square$ Martín D, Schatton D, Wiederstein JL, Marx M, Khiati S, Krüger M et al. CLUH granules coordinate translation of mitochondrial proteins with mTORC1 signaling and mitophagy. EMBO J 2020; 39: e102731.

22 Morita M, Prudent J, Basu K, Goyon V, Katsumura S, Hulea L et al. mTOR Controls Mitochondrial Dynamics and Cell Survival via MTFP1. Mol Cell 2017; 67: 922-935.

23 Clarke K, Ricciardi S, Pearson T, Bharudin I, Davidsen PK, Bonomo M et al. The Role of Eif6 in Skeletal Muscle Homeostasis Revealed by Endurance Training Co-expression Networks. Cell Rep 2017. doi:10.1016/j.celrep.2017.10.040.

24 Biffo S, Manfrini N, Ricciardi S. Crosstalks between translation and metabolism in cancer. Curr. Opin. Genet. Dev. 2018. doi:10.1016/j.gde.2017.10.011.

25 Ricciardi S, Manfrini N, Alfieri R, Calamita P, Crosti MC, Gallo S et al. The Translational Machinery of Human CD4 + T Cells Is Poised for Activation and Controls the Switch from Quiescence to Metabolic Remodeling. Cell Metab 2018; 28: 895-906.e5.

26 Pourdehnad M, Truitt ML, Siddiqi IN, Ducker GS, Shokat KM, Ruggero D. Myc and mTOR converge on a common node in protein synthesis control that confers synthetic lethality in Mycdriven cancers. Proc Natl Acad Sci U S A 2013; 110: 11988-93.

27 Hong S, Freeberg MA, Han T, Kamath A, Yao Y, Fukuda T et al. LARP1 functions as a molecular switch for mTORC1-mediated translation of an essential class of mRNAs. Elife 2017; 6: e25237.

28 Amaldi F, Pierandrei-Amaldi P. TOP genes: a translationally controlled class of genes including those coding for ribosomal proteins. Prog. Mol. Subcell. Biol. 1997; : 1-17.

29 Gressner O, Schilling T, Lorenz K, Schulze Schleithoff E, Koch A, Schulze-Bergkamen H et al. TAp63alpha induces apoptosis by activating signaling via death receptors and mitochondria. Embo $J$ 2005; 24: 2458-2471.

30 Kissil JL, Deiss LP, Bayewitch M, Raveh T, Khaspekov G, Kimchi A. Isolation of DAP3, a novel mediator of interferon- $\gamma$-induced cell death. J Biol Chem 1995; 46: 27932-6.

31 Yoo YA, Kim MJ, Park JK, Chung YM, Lee JH, Chi S-G et al. Mitochondrial Ribosomal Protein L41 Suppresses Cell Growth in Association with p53 and p27Kip1. Mol Cell Biol 2005; 15: 660313.

32 Kim HJ, Maiti P, Barrientos A. Mitochondrial ribosomes in cancer. Semin. Cancer Biol. 2017; : 67-81.

33 Bolger AM, Lohse M, Usadel B. Trimmomatic: a flexible trimmer for Illumina sequence data. Bioinformatics 2014; 30: 2114-2120.

34 Patro R, Duggal G, Love MI, Irizarry RA, Kingsfor C. Salmonprovides accurate, fast, and biasaware transcript expression estimates using dual-phase inference. bioRxiv Prepr 2016.

35 Robinson MD, McCarthy DJ, Smyth GK. edgeR: a Bioconductor package for differential expression analysis of digital gene expression data. Bioinformatics 2010; 26: 139-140.

36 Bailey TL. DREME: Motif discovery in transcription factor ChIP-seq data. Bioinformatics 2011; 15: $1653-1659$.

37 Provenzani A, Fronza R, Loreni F, Pascale A, Amadio M, Quattrone A. Global alterations in mRNA polysomal recruitment in a cell model of colorectal cancer progression to metastasis. Carcinogenesis 2006; 27: 1323-1333.

38 Viero G, Lunelli L, Passerini A, Bianchini P, Gilbert RJ, Bernabò P et al. Three distinct ribosome assemblies modulated by translation are the building blocks of polysomes. J Cell Biol 2015; 208: 581-96.

39 Panda A, Martindale J, Gorospe M. Polysome Fractionation to Analyze mRNA Distribution Profiles. BIO-PROTOCOL 2017; 7: e2126.

40 Belin S, Hacot S, Daudignon L, Therizols G, Pourpe S, Mertani HC et al. Purification of ribosomes from human cell lines. In: Current Protocols in Cell Biology. 2010, p unit 3.40. 
41 Cornella N, Tebaldi T, Gasperini L, Singh J, Padgett RA, Rossi A et al. The hnRNP RALY regulates transcription and cell proliferation by modulating the expression of specific factors including the proliferation marker E2F1. J Biol Chem 2017; 292: 19674-19692.

42 Rossi A, Moro A, Tebaldi T, Cornella N, Gasperini L, Lunelli L et al. Identification and dynamic changes of RNAs isolated from RALY-containing ribonucleoprotein complexes. Nucleic Acids Res 2017; 45: 6775-6792.

Table S1: RNA-seq Fold Change data and results of GSEA analysis for the comparison between HCT_shDHX30 and HCT_shNT.

A), B) ID, gene symbol, fold change, F value, P value and FDR based on RNA-seq data of (A) polysomal RNA, (B) total RNA. GSEA data for polysomal (C) or total (D) RNA-seq data.

\section{Table S2: Primers, probe and siRNAs.}

Contains information on the commercial siRNAs used to silence DHX30 transcript variants and the sequences of all primers used in qRT-PCR experiments.

Table S3: Antibodies.

Lists of primary and secondary antibodies used in a) immunoblots and b) immunofluorescence experiments.

\section{SUPPLEMENTAL FIGURES \& LEGENDS}

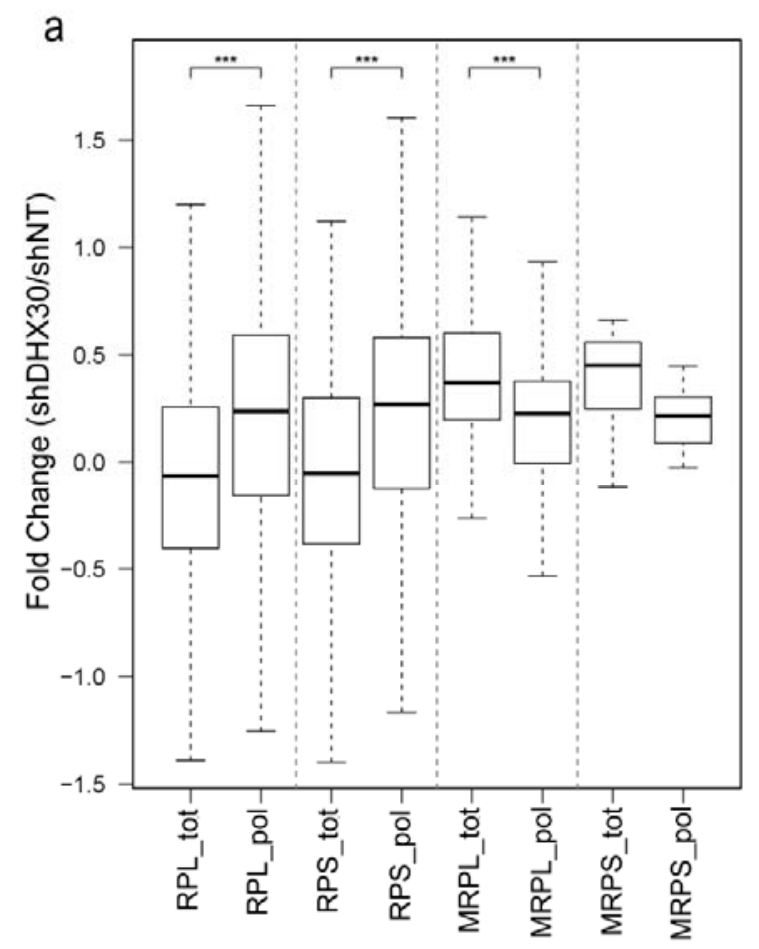

b

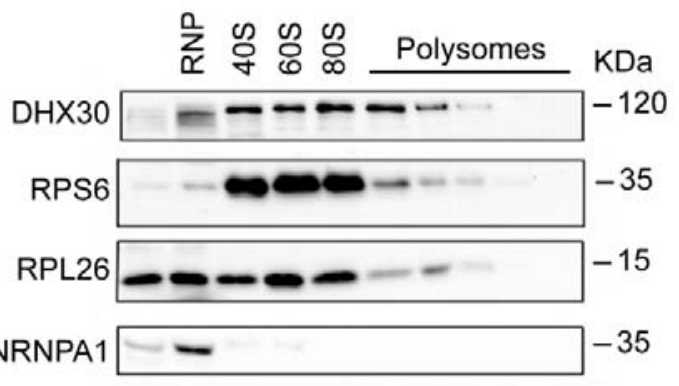

C
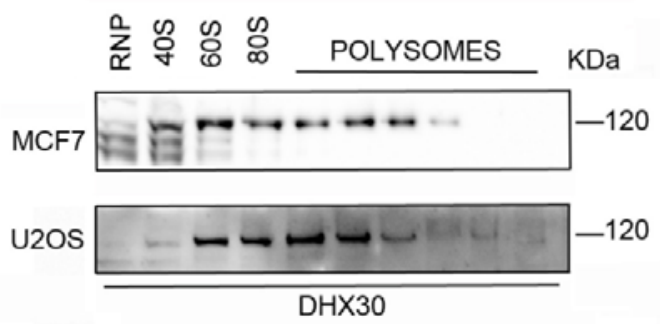

Figure S1. Data supporting Figure 1.

A) DHX30 depletion leads to slight changes in the expression of ribosomal protein genes. Box plot of the expression fold changes in both total and polysomal RNAs for the indicated transcript groups in 
HCT_shDHX30 relative to the HCT116_shNT control. ***p < 0.001. B) Western blot to visualize DHX30 distribution in the different fractions of $\mathrm{HC} 116$ cells after polysome profiling obtained by a linear 15-50\% sucrose gradient. RPS6, RPL26, and HNRNPA1 were used as controls. C) DHX30 protein is associated with ribosomal subunits and polysomes in MCF-7 and U2OS cells. Proteins were extracted from each fraction and subjected to western blot analysis using an anti-DHX30 antibody for immunodetection.

a

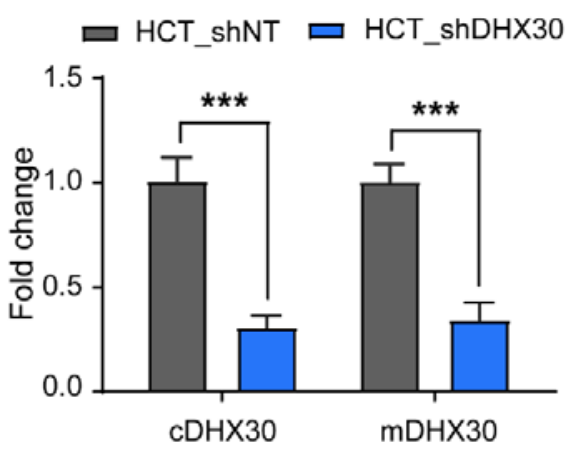

$\mathrm{b}$

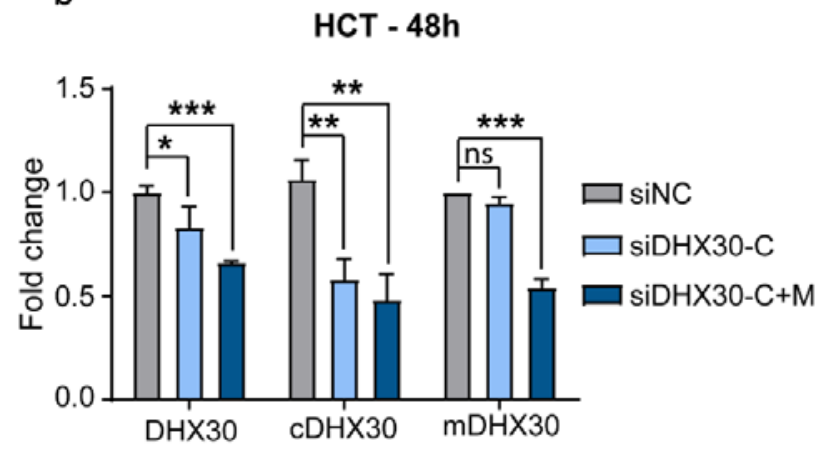

C

HCT- 96h

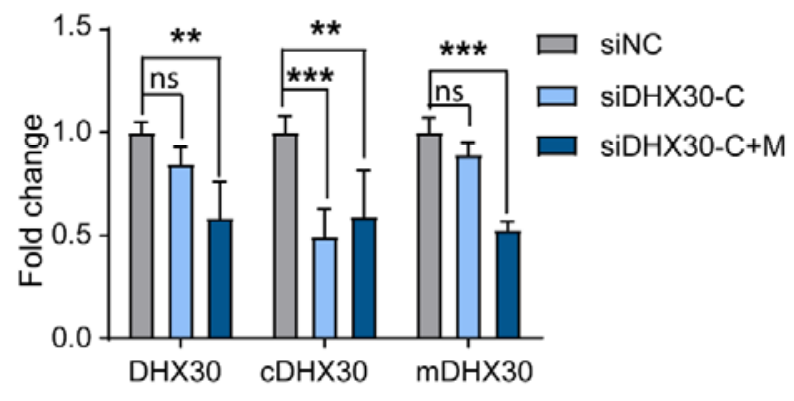

d

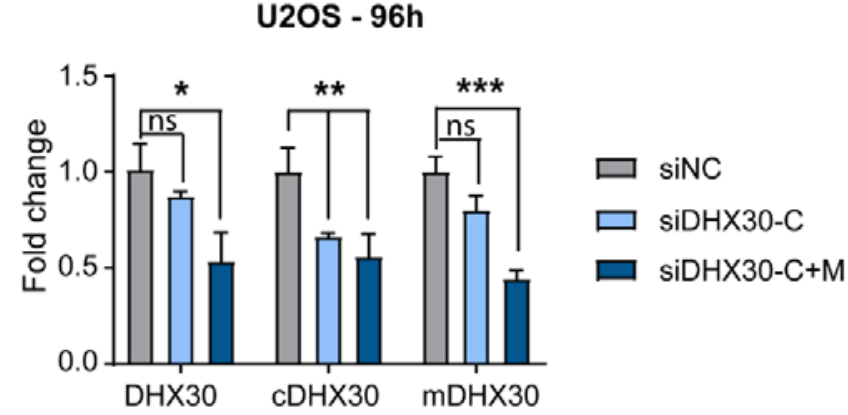

e

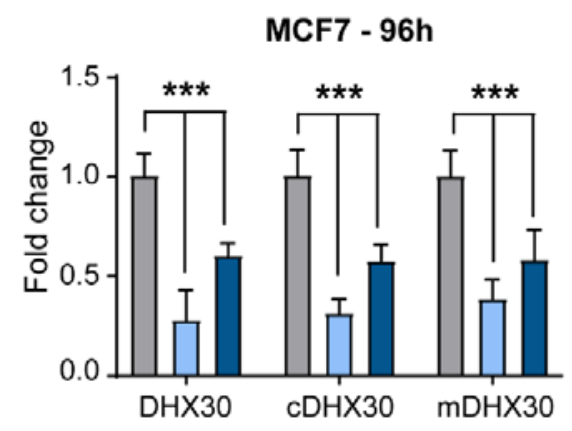

Figure S2. Efficacy of both stable and transient DHX30 silencing in HCT116, MCF-7 and U2OS cells.

A) Relative mRNA levels of cytoplasmic (cDHX30) and mitochondrial (mDHX30) DHX30 transcript variants in HCT116_shDHX30 compared to the shNT control clone. Data are mean $\pm \mathrm{SD}(\mathrm{n}=3)$; ***p < 0.001. B) qRT-PCR to verify the transient silencing of DHX30 in HCT116 using the indicated siRNAs for 48 hours. PCR primers annealing to: (i) a portion of the coding sequence that is present in all transcript variants (DHX30); (ii) the first exon specific of cytoplasmic DHX30 (cDHX30) or (iii) the alternative first exon specific of mitochondrial DHX30 (mDHX30) were used. Data are mean \pm SD $(n=3)$; 
$* \mathrm{p}<0.05, * * \mathrm{p}<0.01 ; * * * \mathrm{p}<0.001$. C) qRT-PCR to verify the silencing of DHX30 in HCT116 96 hours after transient transfections as in B). Data are mean $\pm \mathrm{SD}(\mathrm{n}=3)$; ** $<0.01$; *** $<0.001$. D), E) qRTPCR to verify the efficacy of the transient silencing of DHX30 in U2OS C) or MCF-7 D) using the same primers as in B). Samples were collected for processing 96 hours after silencing with corresponding siRNA. Data are mean $\pm \mathrm{SD}(\mathrm{n}=3) ; * \mathrm{p}<0.05 ; * * \mathrm{p}<0.01 ; * * \mathrm{p}<0.001$. 
a

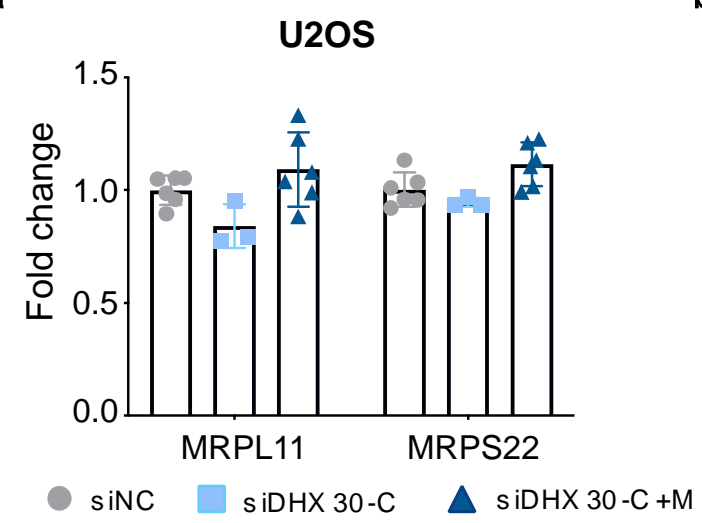

C
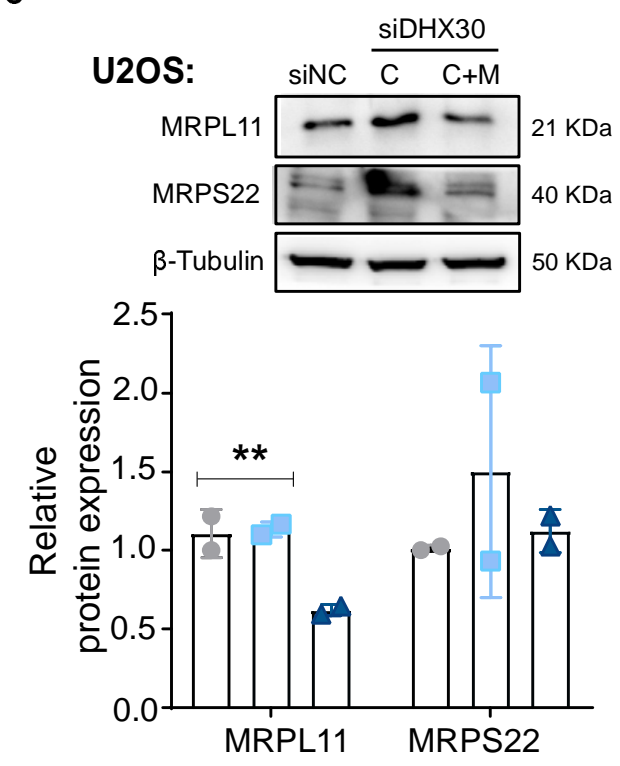

sinc $\square$ siDHX $30-\mathrm{C} \triangle \operatorname{siDHX} 30-\mathrm{C}+\mathrm{M}$ b

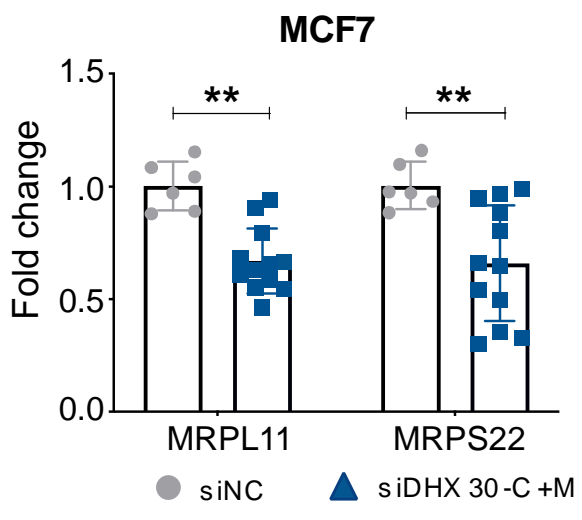

e

MT-ATP6

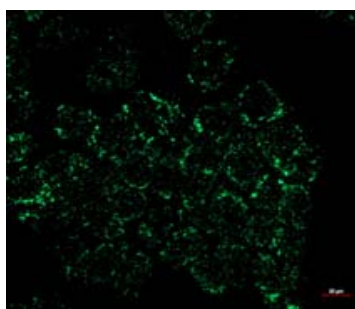

MT-ATP8

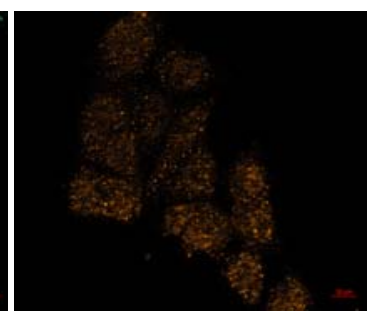

$\mathrm{DH} \times 30$

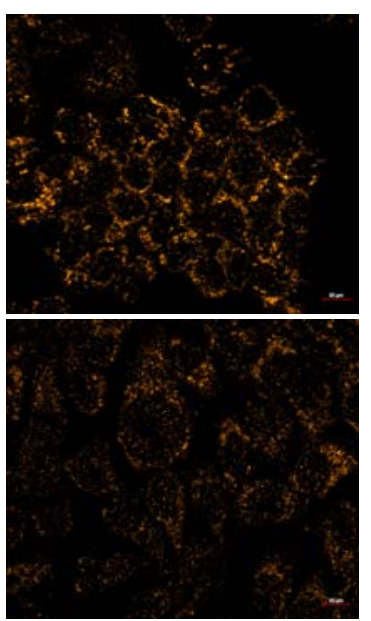


Figure S3. Impact of DHX30 depletion on nuclear encoded mitoribosome components in MCF7 and U2OS, and on mitochondrial OXPHOS components in HCT116 cells.

A) and B) qRT-PCR of MRPL11 and MRPS22 in U2OS (A) and MCF7 (B) cells transiently silenced for cytoplasmic DHX30 (siDHX30-C) or for both cytoplasmic and mitochondrial variants (siDHX30-C+M) for 96 hours. Data are compared to the siRNA negative control ( siNC) and are mean $\pm \mathrm{SD}(\mathrm{n}=3)$; **p < 0.01. C) (Upper panel) representative western blot of MRPL11 and MRPS22 in U2OS silenced transiently as in A). (Lower panel) Relative protein quantification of nuclear encoded mitoribosome components in U2OS transiently silenced for DHX30. Data are mean \pm SD $(n=3) ; * * p<0.01$. D) (Upper panel) representative western blot of MRPL11 and MRPS22 in MCF7 silenced transiently for both cytoplasmic and mitochondrial variants (MCF7_siDHX30-C+M). (Lower panel) Relative protein quantification of nuclear encoded mitoribosome components in MCF7 transiently silenced for DHX30. Data are mean \pm SD $(n=3)$; $* p<0.05$. E) Representative images of one of three independent immunofluorescence experiments to visualize DHX30, MT-ATP8 (red), or MT-ATP6 (green) expression in HCT116_shDHX30 and HCT116_shNT. 
a

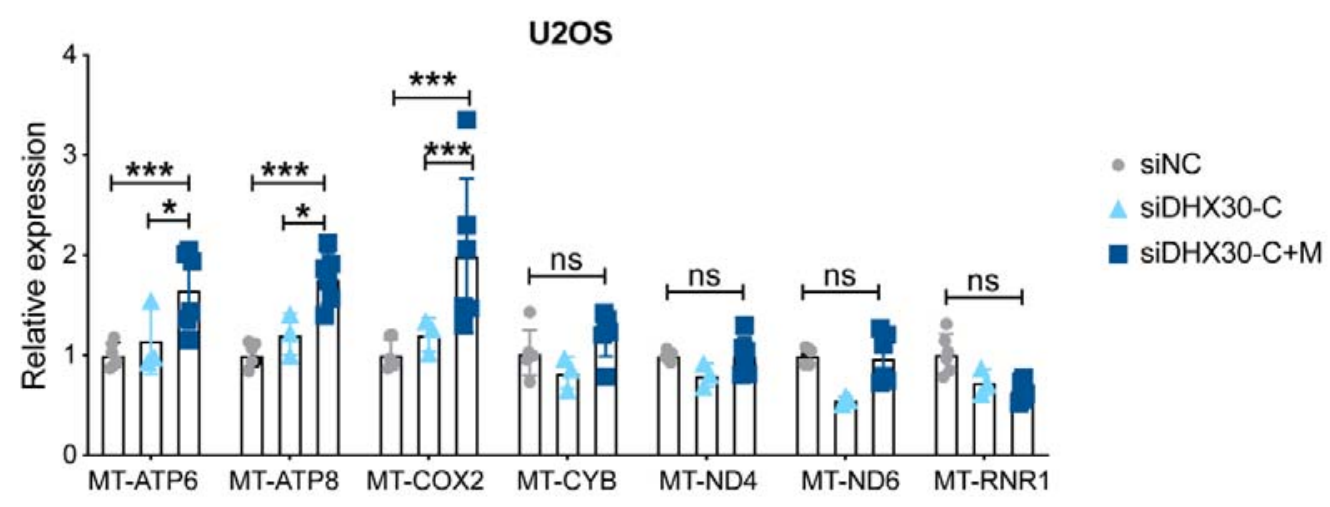

b

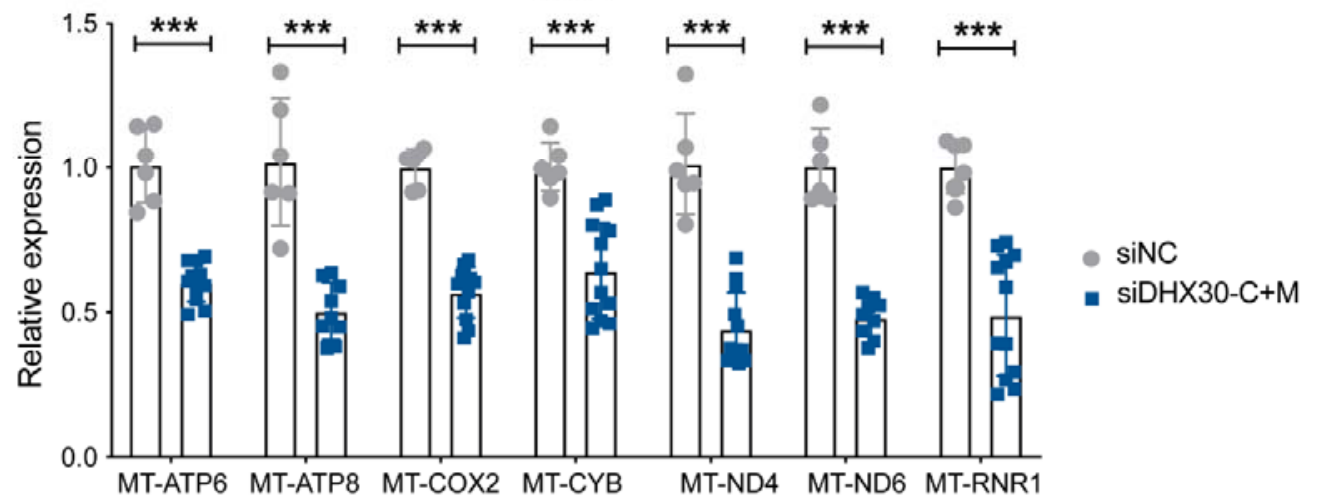

C

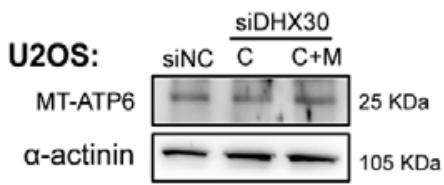

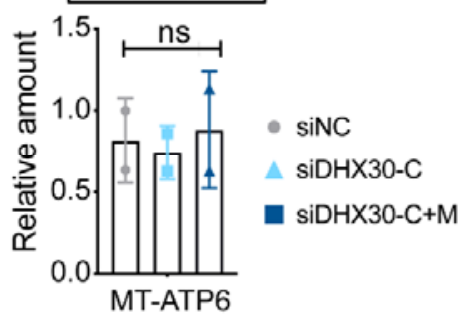

e

U2OS

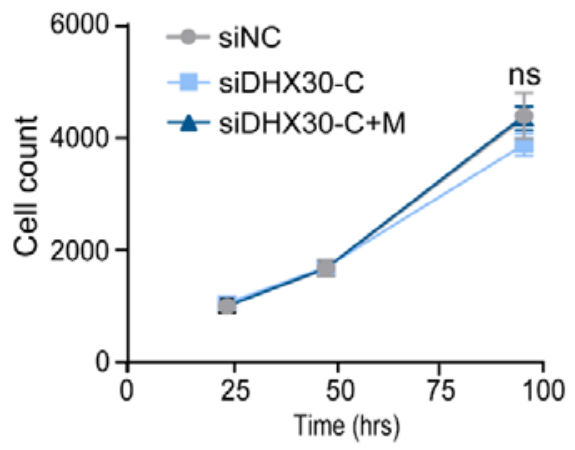

d

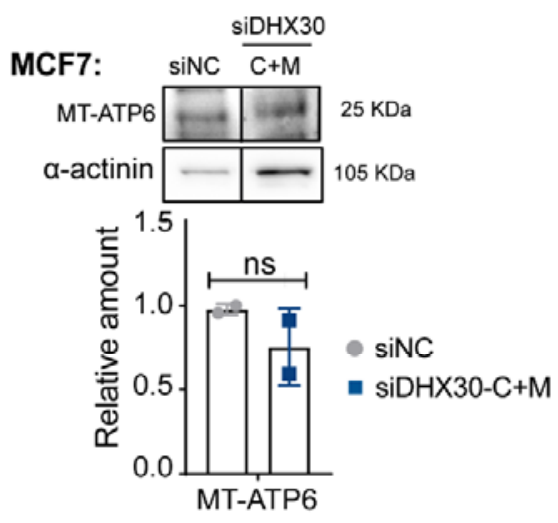

MCF7

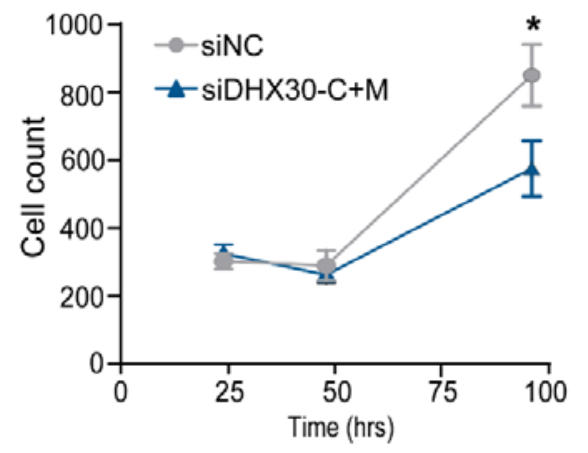


Figure S4. Impact of DHX30 depletion on mitochondrially encoded OXPHOS components and on proliferation in MCF7 and U2OS cells.

A) and B) qRT-PCR of selected mitochondrially encoded genes in U2OS (A)and MCF7 (B) cells transiently silenced for cytoplasmic DHX30 (siDHX30-C) or for both cytoplasmic and mitochondrial variants (siDHX30-C+M) for 96 hours. Data are compared to the siRNA negative control (siNC). Mean and individual points are shown ( $\mathrm{n}=2$ biological replicates); *p $<0.05$; ***p < 0.005 . C) (Up) representative western blot of MT-ATP6 in U2OS transiently silenced as in A). (Down) Relative protein quantification of MT-ATP6 in U2OS. Mean and individual points are shown (n=2). D) (Up) representative western blot of MT-ATP6 in MCF7 transiently silenced for both cytoplasmic and mitochondrial variants (MCF7_siDHX30-C+M). (Down) Relative protein quantification of MT-ATP6. Mean and individual points are shown $(n=2)$. E) Relative cell proliferation measured by high-content microscopy in digital phase contrast in U2OS (Left panel) and MCF7 (Right panel) transiently silenced for DHX30 variants. Data are mean \pm SD ( $n=2$ biological replicates each with three independent wells); $* \mathrm{p}<0.05$. 
bioRxiv preprint doi: https://doi.org/10.1101/2020.07.13.196709; this version posted July 13, 2020. The copyright holder for this preprint (which was not certified by peer review) is the author/funder. All rights reserved. No reuse allowed without permission.

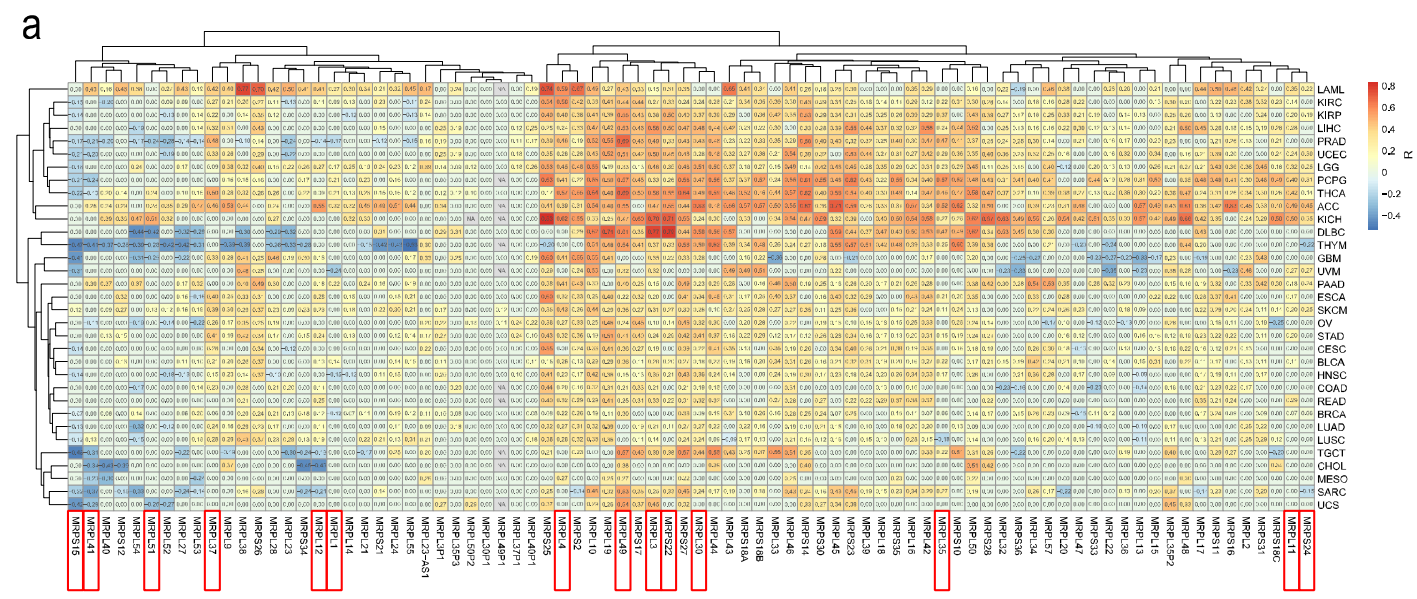

b

\begin{tabular}{|c|c|c|c|c|c|c|c|c|}
\hline \multirow{2}{*}{\multicolumn{2}{|c|}{ see panelA }} & \multicolumn{6}{|c|}{ Data extracted from the GEPIA2 web server ${ }^{\wedge}$} & \multirow[b]{3}{*}{ tumor type name } \\
\hline & & \multicolumn{2}{|c|}{$\begin{array}{l}\text { Expression } \\
\text { Correlation }^{*}\end{array}$} & \multicolumn{4}{|c|}{$\begin{array}{l}\text { Outcome } \\
\end{array}$} & \\
\hline clustering & tumor type & $\mathrm{R}$ & $\mathrm{p}$-value & HR (high) & $\begin{array}{c}\operatorname{Logrank} p \\
\text { value }\end{array}$ & $p(H R)$ & $\mathrm{n}$ & \\
\hline \multirow{11}{*}{ Cluster1 } & LAML & 0.5 & $3.10 \mathrm{E}-12$ & 3.3 & 0.0035 & 0.0055 & 76 & Acute Myeloid Leukemia \\
\hline & KIRC & 0.39 & & 0.69 & 0.017 & 0.017 & 516 & Kidney renal clear cell carcinoma \\
\hline & KIRP & 0.44 & $8.90 \mathrm{E}-15$ & 0.87 & 0.65 & 0.65 & 282 & Kidney renal papillary cell carcinoma \\
\hline & LIHC & 0.49 & & 1.9 & 0.00027 & 0.00035 & 364 & Liver hepatocellular carcinoma \\
\hline & PRAD & 0.44 & & 2.1 & 0.00063 & 0.00083 & 492 & Prostate adenocarcinoma \\
\hline & UCEC & 0.4 & $5.70 \mathrm{E}-11$ & 0.64 & 0.21 & 0.22 & 172 & Uterine Corpus Endometrial Carcinoma \\
\hline & LGG & 0.42 & & 1.7 & 0.0045 & 0.005 & 514 & Brain Lower Grade Glioma \\
\hline & PCPG & 0.52 & $3.60 \mathrm{E}-14$ & 1.2 & 0.74 & 0.74 & 182 & Pheochromocytoma and Paraganglioma \\
\hline & THCA & 0.59 & & 0.66 & 0.16 & 0.16 & 510 & Thyroid carcinoma \\
\hline & ACC & 0.67 & $2.40 \mathrm{E}-11$ & 3.3 & 0.0035 & 0.0055 & 76 & Adrenocortical carcinoma \\
\hline & $\mathrm{KICH}$ & 0.66 & $1.70 \mathrm{E}-09$ & 9.5 & 0.0096 & 0.0034 & 64 & Kidney Chromophobe \\
\hline \multirow{22}{*}{ Cluster 2} & DLBC & 0.37 & $1.00 \mathrm{E}-02$ & 0.65 & 0.56 & 0.56 & 46 & Lymphoid Neoplasm Diffuse Large B-cell Lymphoma \\
\hline & THYM & 0.21 & $2.20 \mathrm{E}-02$ & 1.5 & 0.37 & 0.37 & 119 & Thymoma \\
\hline & GBM & 0.13 & $9.30 \mathrm{E}-02$ & 0.98 & 0.86 & 0.9 & 162 & Glioblastoma multiforme \\
\hline & UVM & 0.04 & $7.30 \mathrm{E}-01$ & 3 & 0.025 & 0.033 & 78 & Uveal Melanoma \\
\hline & PAAD & 0.41 & $1.70 \mathrm{E}-08$ & 1.3 & 0.21 & 0.22 & 178 & Pancreatic adenocarcinoma \\
\hline & ESCA & 0.31 & $2.30 \mathrm{E}-05$ & 1 & 0.99 & 1 & 182 & Esophageal carcinoma \\
\hline & SKCM & 0.44 & $0.00 \mathrm{E}+00$ & 1.4 & 0.027 & 0.028 & 458 & Skin Cutaneous Melanoma \\
\hline & OV & 0.17 & $3.70 \mathrm{E}-04$ & 0.85 & 0.18 & 0.19 & 424 & Ovarian serous cystadenocarcinoma \\
\hline & STAD & 0.35 & $3.00 \mathrm{E}-13$ & 0.82 & 0.21 & 0.22 & 384 & Stomach adenocarcinoma \\
\hline & CESC & 0.21 & $3.10 \mathrm{E}-04$ & 1.1 & 0.75 & 0.75 & 292 & Cervical squamous cell carcinoma and endocervical adenocarcinoma \\
\hline & BLCA & 0.31 & $2.80 \mathrm{E}-10$ & 1.1 & 0.63 & 0.63 & 402 & Bladder Urothelial Carcinoma \\
\hline & HNSC & 0.22 & $2.70 \mathrm{E}-07$ & 1.1 & 0.32 & 0.33 & 518 & Head and Neck squamous cell carcinoma \\
\hline & COAD & 0.17 & $4.90 \mathrm{E}-03$ & 0.79 & 0.33 & 0.34 & 270 & Colon adenocarcinoma \\
\hline & READ & 0.37 & $2.80 \mathrm{E}-04$ & 0.74 & 0.53 & 0.53 & 92 & Rectum adenocarcinoma \\
\hline & BRCA & 0.22 & $1.70 \mathrm{E}-13$ & 1.3 & 0.13 & 0.13 & 1070 & Breast invasive carcinoma \\
\hline & DUAD & 0.16 & $6.10 \mathrm{E}-04$ & 1.6 & 0.0027 & 0.003 & 478 & Lung adenocarcinoma \\
\hline & LUSC & 0.2 & $6.80 \mathrm{E}-06$ & 0.86 & 0.27 & 0.27 & 482 & Lung squamous cell carcinoma \\
\hline & TGCT & 0.17 & $5.40 \mathrm{E}-02$ & 1.4 & 0.34 & 0.34 & 136 & Testicular Germ Cell Tumors \\
\hline & $\mathrm{CHOL}$ & -0.16 & $3.70 \mathrm{E}-01$ & 1.1 .1 & 0.79 & 0.8 & $\frac{136}{36}$ & Cholangio carcinoma \\
\hline & MESO & 0.05 & $6.60 \mathrm{E}-01$ & 1.5 & 0.097 & 0.098 & 82 & Mesothelioma \\
\hline & SARC & 0.12 & $6.20 \mathrm{E}-02$ & 1.3 & 0.25 & 0.25 & 262 & Sarcoma \\
\hline & & & & 1.2 & 0.54 & 0.53 & 56 & Uterine Carcinosarcoma \\
\hline \multicolumn{9}{|c|}{ ^ http://gepia2.cancer-pku.cn/\#index } \\
\hline
\end{tabular}

C

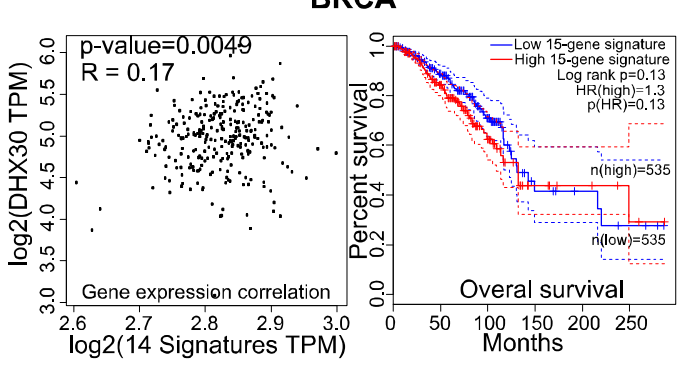

d

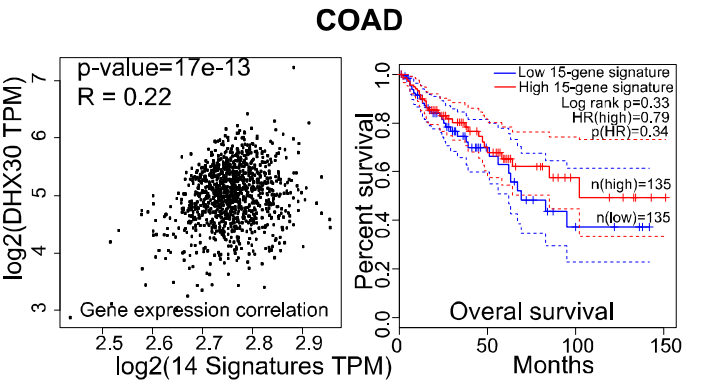

Figure S5. DHX30 expression positively correlates with the expression of mitoribosomal protein transcripts and can have prognostic significance. 
A) Pearson correlation between the expression of DHX30 and each mitoribosomal protein transcript in cancer samples from TGCA. Data were extracted from the GEPIA web server (see methods for details). Unsupervised clustering revealed two major clusters based on the level of gene expression correlation and two main clusters among cancer types. Boxed in red are the names of the fourteen transcripts that are candidate DHX30 direct targets (see text for details and panel) B) The expression correlation data (R value and $\mathrm{p}$ value) between DHX30 and the combined group of 14 mitoribosomal protein transcripts is listed for each cancer type presented in the same order as for panel A). Data from Kaplan-Meier survival analysis for the aggregated 15-gene signature (DHX30 + the 14 mitoribosomal protein transcripts). Significant differences are highlighted in bold font. C), D) Correlation and Overall Survival KaplanMeier curves for two negative examples from cluster 2; colon adenocarcinoma (COAD); breast cancer (BRCA). As for panel B), Hazardous Ratio for the high expression group, p values, and sample size are indicated. 


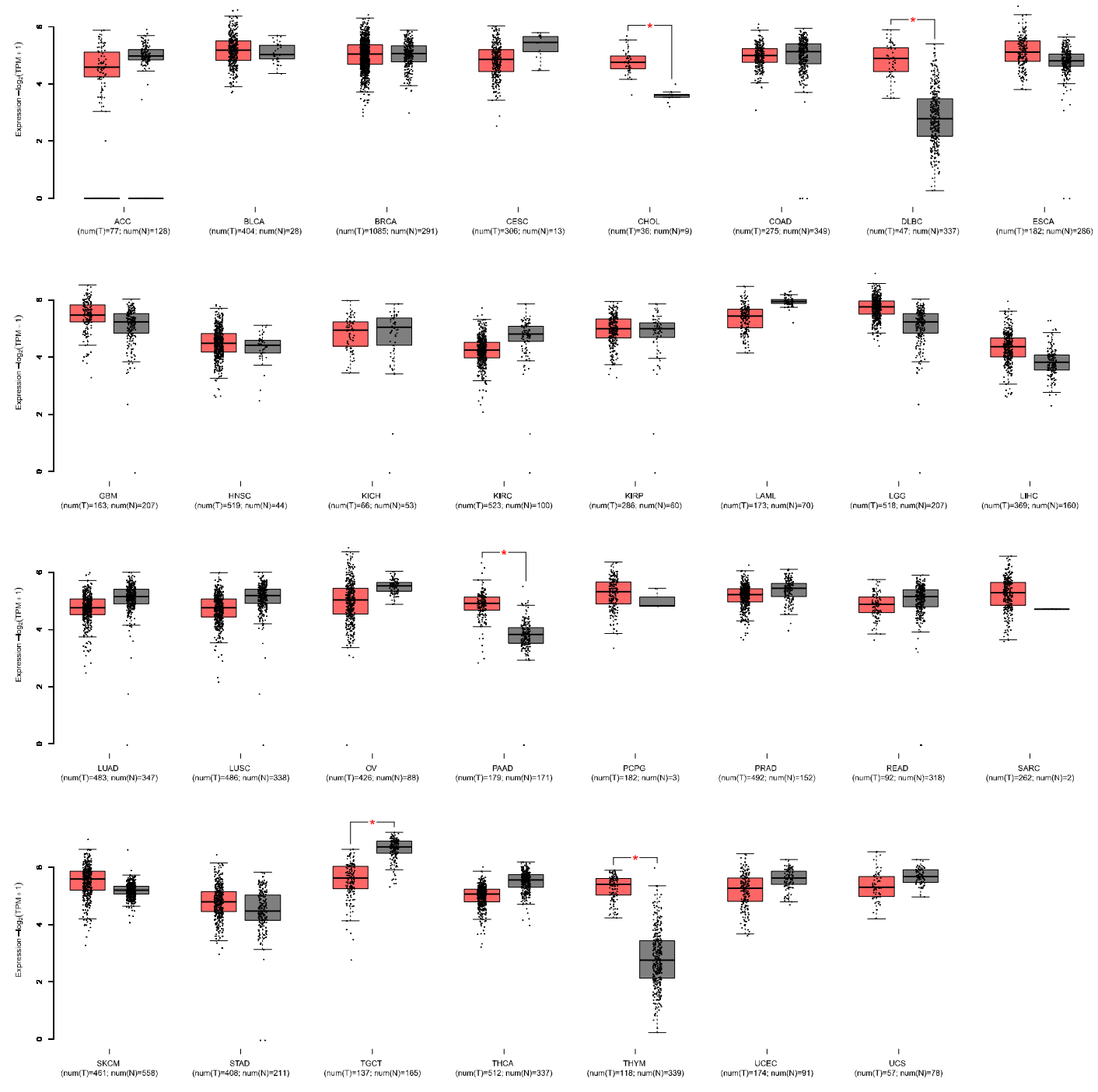

Figure S6. Expression of DHX30 in TCGA tumor types and matched healthy tissues. Each boxplot pair displays the expression levels of DHX30 in a TCGA tumor samples (red box) and in the corresponding healthy tissue samples (grey box, composed of TCGA normal samples and GTEX samples of the same tissue). Expression is displayed in $\log 2(\operatorname{tags}$ per million sequenced reads +1$)$. Plots were obtained with GEPIA2. Significance of the difference between tumor and normal samples is indicated by a red asterisk and represents a $\mathrm{q}$-value $<=0.01$ and a $|\log 2 \mathrm{FC}|>1$ as computed in GEPIA 2 by the ANOVA method. 
GTEX

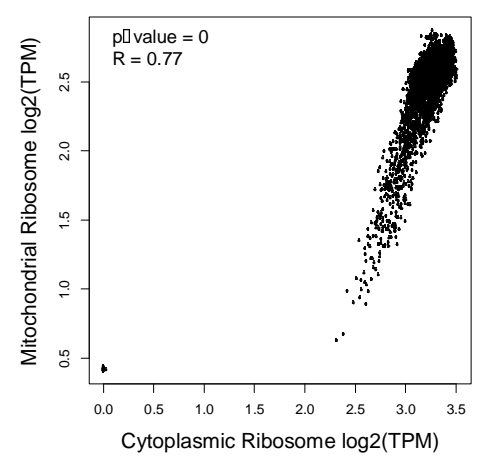

TCGA normal

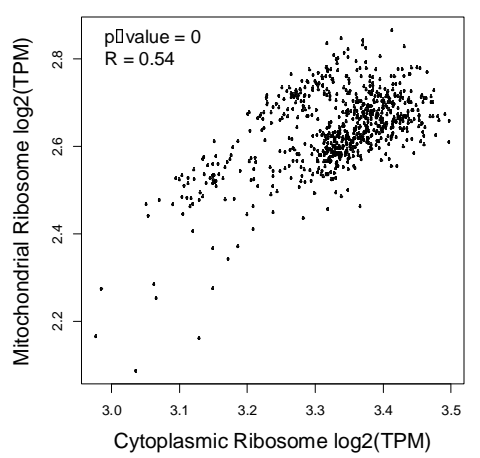

TCGA tumor

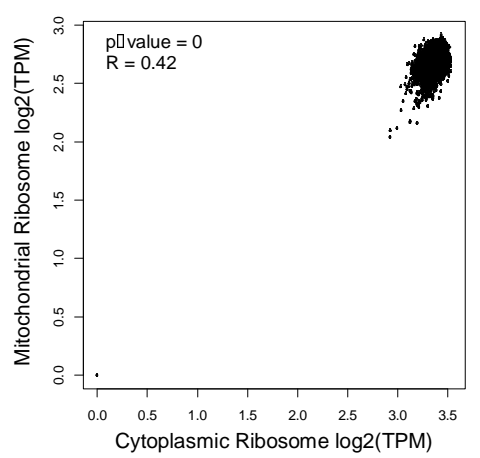

Figure S7. Expression correlation of cytoplasmic and mitochondrial ribosome component genes in TCGA and GTEX samples. The three scatterplots display the Pearson correlation ( $R$ and $p$-value indicated in the upper left corner of each plot) between the expression (indicated as log2(tags per million sequenced reads)) of cytoplasmic ribosome component genes ( $\mathrm{X}$ axis) and mitochondrial ribosome component genes ( $\mathrm{Y}$ axis). The leftmost plot shows the correlation in GTEX healthy tissues samples, the center plot presents the correlation in TCGA normal samples, and the rightmost plot shows the correlation in TCGA tumor samples. Plots were obtained with GEPIA2. 


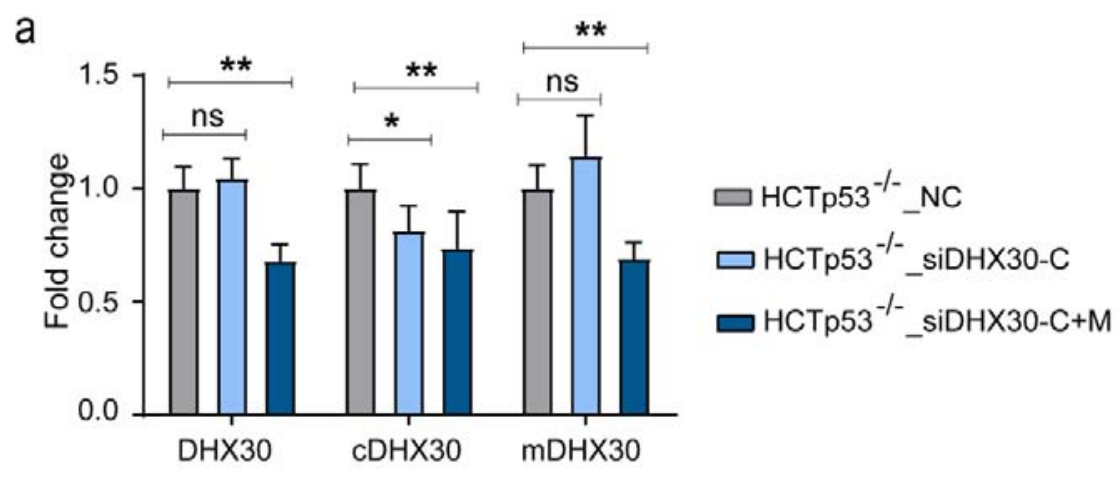

b
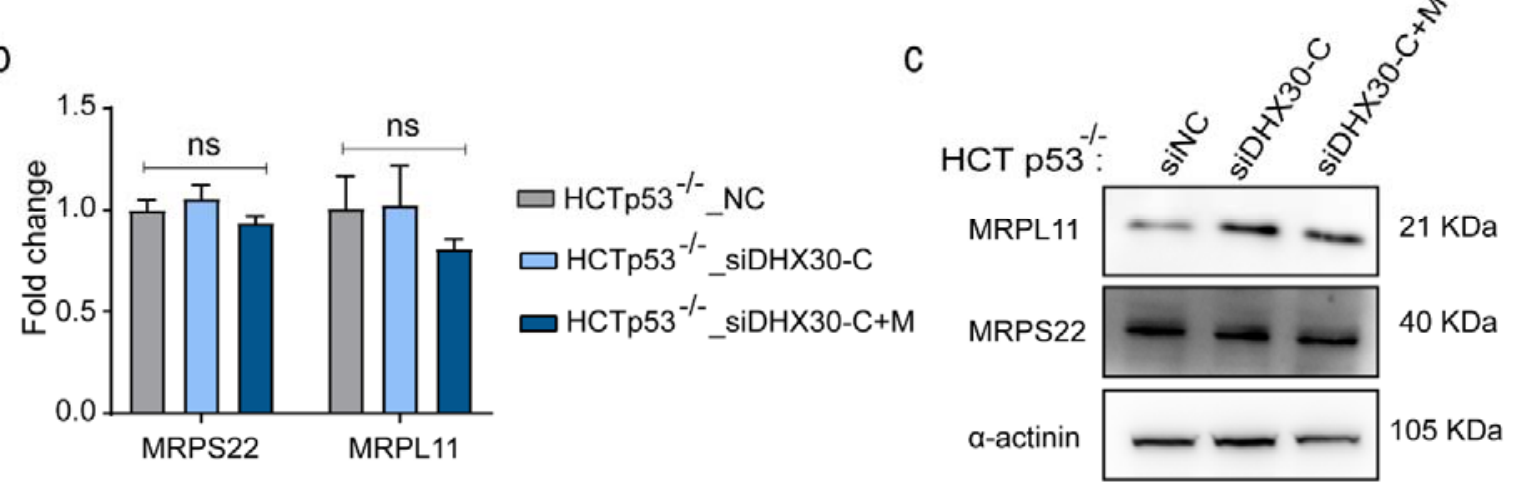

d

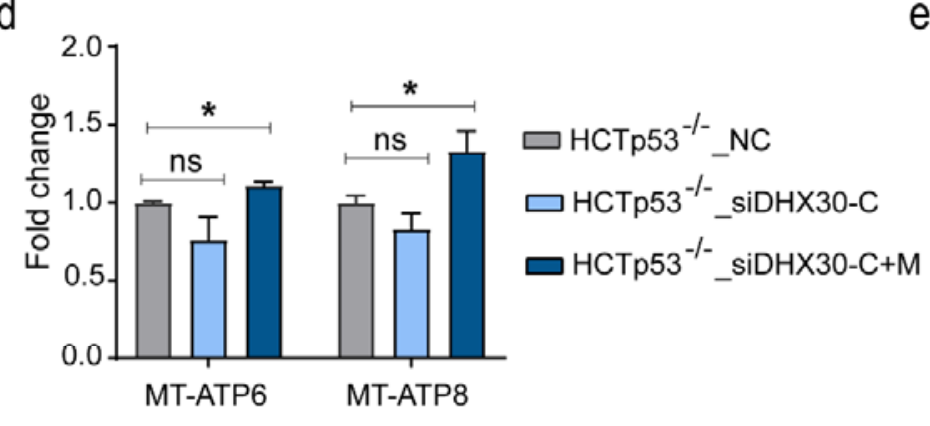

e

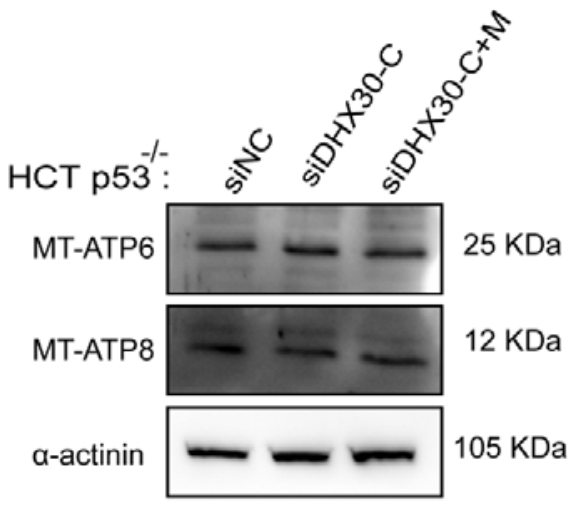

f

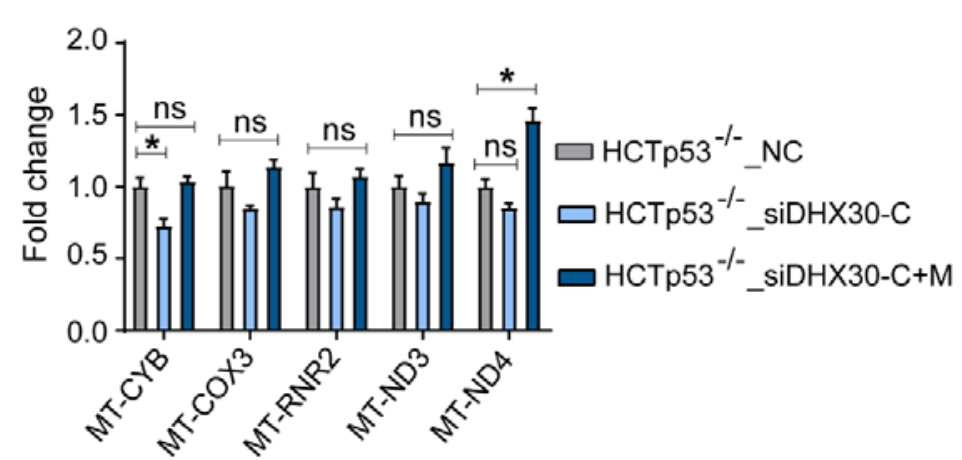

g

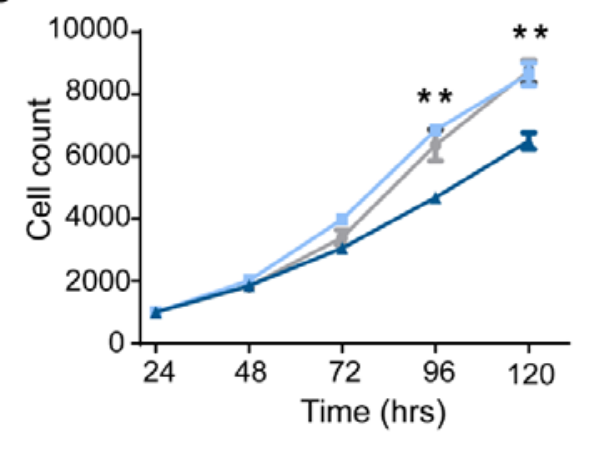

Figure S8. Silencing of DHX30 does not affect mitoribosome and OXPHOS components in HCT116 when $\mathrm{p} 53$ is knock-out.

A) qRT-PCR to verify the transient silencing of DHX30 in HCT116 $\mathrm{p} 53^{-/-}$using primers annealing to: (i) a portion of the coding sequence that is present in all transcript variants (tDHX30); (ii) the first 
exon specific of cytoplasmic DHX30 (cDHX30) or (iii) the alternative first exon specific of mitochondrial DHX30 (mDHX30). Experiment was performed 96 hours post silencing. B), C) Relative mRNA B) and protein C) levels of MRPL11 and MRPS22 in HCT116 p53 $3^{-/-}$transiently silenced as shown in A) and compared to the siNC control. $\alpha$-actinin was used as loading control. Immunoblots represent one of three independent experiments. D), E) Relative mRNA D) and protein E) levels of MT_ATP6 and MT-ATP8 in $\mathrm{HCT} 116{\mathrm{p} 53^{--}}^{-}$transiently silenced as shown in A) and compared to the siNC control. $\alpha$-actinin was used as loading control. Immunoblots represent one of three independent experiments. F) RT-qPCR to study some of mitochondrially encoded OXPHOS components in HCT116 p53-/- transiently silenced for

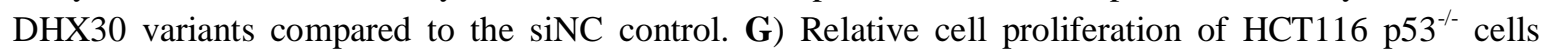
measured by high-content microscopy in digital phase contrast and compared to siNC control at indicated time points. For all panels, data are mean $\pm \mathrm{SD}(\mathrm{n}=3) ;{ }^{*} \mathrm{p}<0.05 ; * * \mathrm{p}<0.01$.

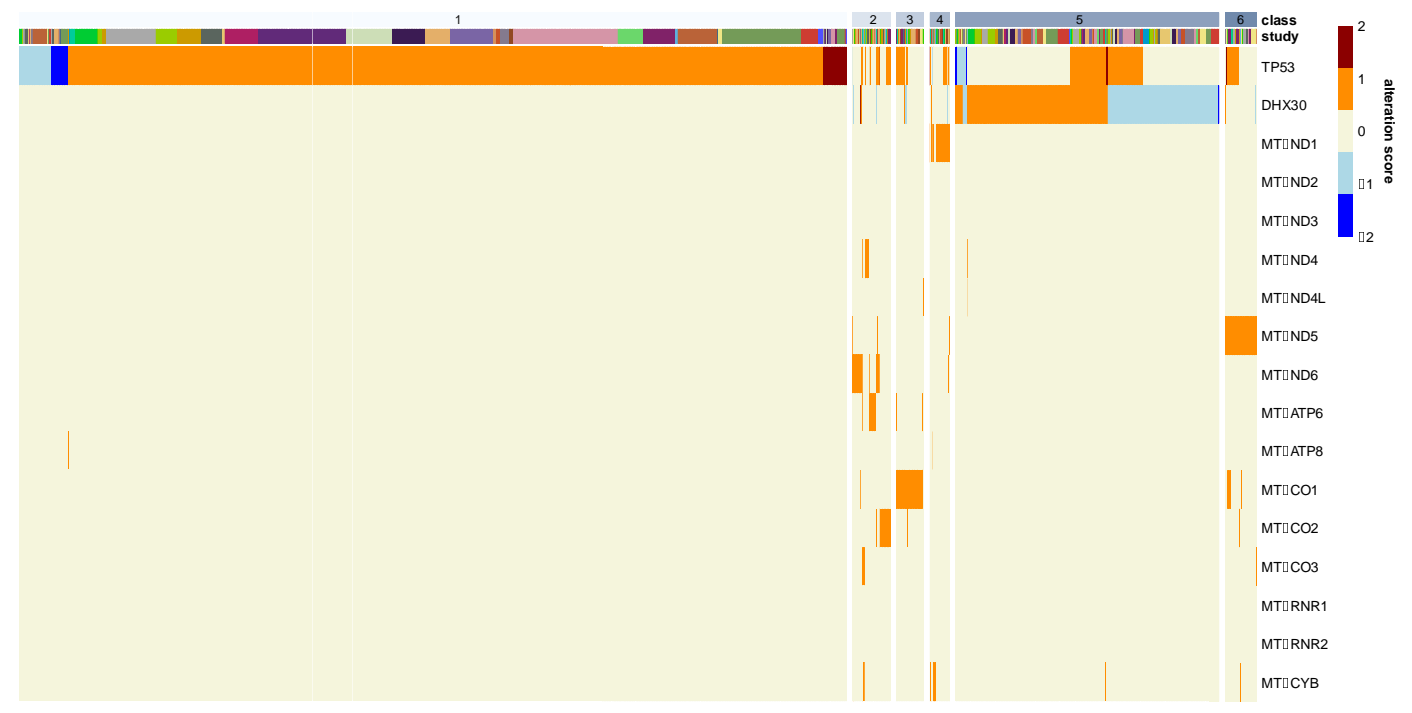

Figure S9. DHX30 copy number alterations in human cancer.

Pan cancer data from TGCA was interrogated for copy number alterations of DHX30, TP53 and mitochondrially encoded genes. A low frequency of DHX30 copy number gain or loss was apparent, and did not appear to be associated with a specific cancer type. No apparent correlation between DHX30 and TP53 copy number status was apparent. Instead, although limited by the low number of occurrences, alterations in mitochondrial genome and DHX30 CNVs seemed to be mutually exclusive. 